\title{
Ueber das Minimum oder Maximum der Potenz- summe der Abstände eines Punktes von gegebenen Punkten, Geraden oder Ebenen.
}

\author{
(Von Herrn Franz Wetzig zu Leipzig.)
}

\section{Ueber das Minimum der Quadratsumme der Abstände eines Punlites von gegebenen Punkten.}

S. 1.

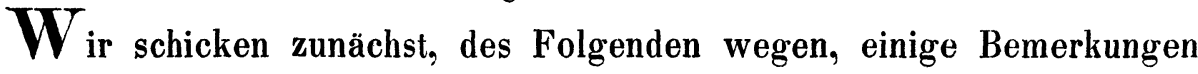
über den Punkt voraus, für welchen die Summe der Quadrate seiner Abstände von gegebenen Punkten ein Minimum ist, und welcher bekanntlich der Schwerpunkt der letzteren ist ${ }^{*}$ ).

Sind $k$ Punkte $A$ gegeben, sind $O$ und $O^{\prime}$ zwei beliebige Punkte, und bezeichnet man die Abstände $O O^{\prime}$ mit $t, O A$ mit $r$ und $O^{\prime} A$ mit $r^{\prime}$ und den Winkel zwischen zwei Geraden $a$ und $b$ einfach durch $a b$, so besteht die Gleichung

$$
r^{\prime 2}=r^{2}+t^{2}-2 r t \cos r t
$$

Bildet man diesen Ausdruck für jeden der Punkte $A$ und summirt, was durch das Zeichen $\Sigma$ ausgedrückt werde, so erhält man

$$
\Sigma r^{\prime 2}=\Sigma r^{2}+k t^{2}-2 t \Sigma r \cos r t .
$$

Wählt man den Punkt $O$ so, dass in Bezug auf jede durch ihn gelegte Gerade die Summe $\Sigma r \cos r t$ verschwindet, dass daher die Abstände $r$, der Grösse und Richtung nach an einander gesetzt, ein geschlossenes Vieleck bilden, so wird

Hieraus folgert man:

$$
\Sigma r^{\prime 2}=\Sigma r^{2}+k t^{2} \text {. }
$$

In Bezug auf den der Bedingung $\Sigma r \cos r t=0$ genügenden Punkt $O$ ist $\sum r^{2}$ ein Minimum.

Dieser Punkt ist der Schwerpunkt der gleichschweren Punkte $A$; denn nimmt man statt $t$ drei auf einander senkrechte Axen und nennt $x, y, z$ die ihnen parallelen Coordinaten von $A$, so folgt $\Sigma x=\Sigma y=\Sigma z=0$.

- ${ }^{*}$ s. Carnot, Geometrie der Stellung Art. 275̄ L'huilier, de maximis et minimis §. $68-78$. 
Der Ort der Punkte $O^{\prime}$, für welche die Quadratsumme der Abstände, $\Sigma r^{\prime 2}$, constant ist, ist eine Kugelfläche (in der Ebene ein Kreis) mit dem Mittelpunkt $O$, und die Zunahme jener Summe ist proportional dem Quadrate des Halbmessers.

Die rechtwinklige Projection des Punktes $O$ auf eine gegebene Gerade oder Ebene ist derjenige Punkt dieser Geraden oder Ebene, für welchen die Quadratsumme der Abstände von den gegebenen Punkten ein Minimum ist; denn jeder andere Punkt dieser Ebene (Geraden) gehört einer Kugel (Kreislinie) constanter Quadratsumme von grösserem Halbmesser an. Diese Projection ist zugleich Mittelpunkt der Kreise der Ebene, oder je zweier Punkte der Geraden, für welche die Quadratsumme constant ist.

\title{
II. Ueber das Minimum der Quadratsumme der Abstände eines Punktes von gegebenen Geraden in der Ebene oder Ebenen im Raume.
}

\author{
S. 2 .
}

1. Sind $k$ Gerade in der Ebene oder $k$ Ebenen im Raume gegeben, und bezeichnet man den senkrechten Abstand eines Punktes $O$ von einer derselben mit $r$, den eines anderen Punktes $O^{\prime}$, in der Entfernung $O O^{\prime}=t$, mit $r^{\prime}$, so hat man, wenn $O$ und $O^{\prime}$ auf derselben Seite der Geraden oder Ebene liegen,

$$
r^{\prime}=r-t \cos r t,
$$

wenn auf entgegerigesetzter,

$$
r^{\prime}=t \cos r t-r .
$$

Man kann daher die erste Gleichung zu Grunde legen mit Beachtung der Regel, dass $r^{\prime}$ das Zeichen wechselt, wenn es auf die entgegengesetzte Seite der Geraden oder Ebene übergeht. Durch Quadrirung folgt

$$
r^{\prime 2}=r^{2}-2 r t \cos r t+t^{2} \cos ^{2} r t .
$$

Bildet man die Summe dieser Quadrate für alle Geraden oder Ebenen, so erhält man

$$
\Sigma r^{\prime 2}=\Sigma r^{2}-2 t \Sigma r \cos r t+t^{2} \Sigma \cos ^{2} r t \text {. }
$$

Wenn daher der Punkt $O$ ein solcher ist, dass in Bezug auf jede durch ihn gelegte Gerade die, Bedingung

$$
\Sigma r \cos r t=0
$$

erfüllt wird, dass also die Abstände $r$, der Grösse und Richtung nach an- 
einander gesetzt, ein geschlossenes Vieleck bilden, so ist

$$
\Sigma r^{2}=\Sigma r^{2}+t^{2} \Sigma \cos ^{2} r t \text {. }
$$

Da aber $t^{2} \Sigma \cos ^{2} r t$ stets positiv ist, so ist $\Sigma r^{2}$ ein Minimum, also der Punkt $O$, für den, bei jeder Lage von $t, \Sigma r \cos r t=0$ ist, der Minimumpunkt.

Hieraus folgt, dass $O$ zugleich der Schwerpunkt der Fusspunkte der Abstände $r$ ist; denn die Bedingungsgleichung $\Sigma r \cos r t=0$ ist dieselbe, wie die im vorigen Paragraphen entwickelte für den Schwerpunkt.

2. Für denselben Punkt $O$ ist bei Geraden in der Ebene auch die Quadratsumme der nicht rechtwinklig, sondern unter einem beliebigen Winkel $u$ genommenen Abstände $\varrho$ ein Minimum, und $O$ ist der Schwerpunkt ihrer Endpunkte auf den Geraden. Denn mit $\Sigma r^{2}$ ist auch $\Sigma \varphi^{2}=\frac{\Sigma r^{2}}{\cos ^{2} u}$ ein Minimum; und bildet die durch $O$ gelegte Gerade $\tau$ mit $t$ ebenfalls den Winkel $u$, so ist mit $\Sigma r \cos r t=0$ die Gleichung $\Sigma \varrho \cos \varrho \tau=0$ identisch. Dreht man also das System der gegebenen Geraden in seiner Ebene um den Punkt $O$, so wird es von dem festen Strahlenbüschel der $r$ beständig in Punkten geschnitten, deren Schwerpunkt $O$ ist.

3. Bei gegebenen Geraden ist das Vieleck, welches die $r$, der Grösse und Richtung nach an einander gesetzt, bilden, mit dem gegebenen gleichwinklig, bei drei Geraden ihm daher ähnlich, woraus folgt, dass im Dreieck die Abstände des Punktes $O$ den zugehörigen Seiten proportional sind. Bei vier Geraden erfordert die Aehnlichkeit eine Bedingungsgleichung (s. S. 8). Giebt es überhaupt in eineın Vieleck einen Punkt, dessen Abstände den zugehörigen Seiten proportional sind, so ist er Minimumpunkt. In jedem regelmässigen Vieleck ist er daher der Mittelpunkt.

\section{S. 3.}

1. Ist $O$ der Punkt einer der gegebenen Geraden oder Ebenen, für welchen die Quadratsumme der Abstände von den übrigen Geraden oder Ebenen ein Minimum ist, so muss in Bezug auf jene Gerade $t$ oder in Bezug auf jede Gerade $t$ jener Ébene die Bedingung $\Sigma r \cos r t=0$ erfüllt werden; setzt man also die $r$ der Grösse und Richtung nach an einander, so müssen Anfangs - und Endpunkt in einer Normalen zur Geraden oder Ebene liegen.

2. Die Punkte paralleler Geraden, für welche die Quadratsumme der Abstände von Geraden in der Ebene ein Minimum, also $\Sigma r \cos r t=0$ ist. 
liegen auf einer gewissen Geraden. Denn ist $O$ der Minimumpunkt der Geraden $t$ in Bezug auf die übrigen Geraden, also $\Sigma r \cos r t=0$, und hat auf einer beliebigen Parallelen $t^{\prime}$ zu $t$, im senkrechten Abstande $y$, ein Punkt $O^{\prime}$ die rechtwinkligen Coordinaten $x$ und $y$, deren Anfangspunkt $O$ ist, so ist, wenn $r t=\alpha$ gesetzt wird,

$$
\begin{gathered}
r=r^{\prime}+y \sin \alpha+x \cos \alpha, \\
\Sigma r \cos \alpha=\Sigma r^{\prime} \cos \alpha+y \Sigma \sin \alpha \cos \alpha+x \Sigma \cos ^{2} \alpha .
\end{gathered}
$$

Da aber $\Sigma r \cos \alpha=0$ ist, so liefert die Bedingung, dass $O^{\prime}$ Minimumpunkt auf $t^{\prime}$, also $\Sigma r^{\prime} \cos \alpha=\Sigma r^{\prime} \cos r^{\prime} t^{\prime}=0$ sein soll, die Gleichung einer Geraden, nämlich

$$
x \Sigma \cos ^{2} \alpha+y \Sigma \sin \alpha \cos \alpha=0 .
$$

3. Die Punkte paralleler Ebenen, für welche die Quadratsumme der Abstände von gegebenen Ebenen ein Minimum ist, liegen gleichfalls auf einer gewissen Geraden. Denn legt man durch den Minimumpunkt $O$ einer Ebene drei rechtwinklige Axen $O X, O Y, O Z$, von denen $O X$ und $O Y$ in dieser Ebene liegen, und bildet $r$ mit ihnen die Winkel $\alpha, \beta, \gamma$, so ist $\Sigma r \cos \alpha=\Sigma r \cos \beta=0$. Hat in einer parallelen Ebene im Abstande z ein Punkt $O^{\prime}$ die Coordinaten $x, y, z$, so ist

$$
r=r^{\prime}+x \cos \alpha+y \cos \beta+z \cos \gamma .
$$

Multiplicirt man diese Gleichung mit $\cos \alpha$, ein zweites Mal mit $\cos \beta$, summirt und berücksichtigt in den beiden erhaltenen Summengleichungen die Bedingungen, dass $O^{\prime}$ Minimumpunkt seiner Ebene sein soll, nämlich $\Sigma r^{\prime} \cos \alpha=\Sigma r^{\prime} \cos \beta=0$, so folgt

$$
\begin{aligned}
& 0=x \Sigma \cos ^{2} \alpha+y \Sigma \cos \alpha \cos \beta+z \Sigma \cos \alpha \cos \gamma, \\
& 0=x \Sigma \cos \alpha \cos \beta+y \Sigma \cos ^{2} \beta+z \Sigma \cos \beta \cos \gamma,
\end{aligned}
$$

wodurch eine Gerade bestimmt wird.

\section{\$. 4.}

Mit Hülfe der Sätze des vorigen Paragraphen lässt sich der Punkt $O$ für beliebig viele Gerade in der Ebene construiren.

1. Sind drei Gerade $a_{1}, a_{2}, a_{3}$ gegeben, welche auf einander die Abschnitte $A_{2} A_{3}=a_{1}, A_{3} A_{1}=a_{2}, A_{1} A_{2}=a_{3}$ bilden, und ist $O_{1}$ der Punkt der Geraden $a_{1}$, für den die Quadratsumme $r_{2}^{2}+r_{3}^{2}$ der Abstände von $a_{2}$ und $a_{3}$ ein Minimum ist, so ist $r_{3} \cos r_{3} a_{1}+r_{2} \cos r_{2} a_{1}=0$, oder $r_{3} \sin A_{2}=r_{2} \sin A_{3}$, 
folglich

$$
\begin{gathered}
r_{2}: r_{3}=\sin A_{2}: \sin A_{3}=a_{2}: a_{3}, \\
O_{1} A_{3}: O_{1} A_{2}=\frac{r_{2}}{\sin A_{3}}: \frac{r_{3}}{\sin A_{2}}=a_{2}^{2}: a_{3}^{2} .
\end{gathered}
$$

Es verhalten sich also die Abstände des Punktes $O_{1}$ wie die zugehörigen Dreiecksseiten und die Abschnitte, die $O_{1}$ auf $a_{1}$ bildet, wie deren Quadrate. Man kann daher $O_{1}$ construiren, indem man innerhalb des Winkels $A_{1}$ zwei Parallelen zu $a_{2}$ und $a_{3}$ in den senkrechten Abständen $a_{2}$ und $a_{3}$ zieht; die Verbindungslinie ihres Durchschnittes mit $A_{1}$ giebt die Gerade $A_{1} O_{1}$.

Sind nun $r_{1}, r_{2}, r_{3}$ die Abstände irgend eines Punktes von $a_{1}, a_{2}, a_{3}$, ferner $\alpha_{1}, \alpha_{2}, \alpha_{3}$ die Winkel, welche $r_{1}, r_{2}, r_{3}$ mit einer gewissen Geraden bilden, so gilt, wenn als solche $a_{1}$ oder eine zu $a_{1}$ Parallele genommen wird, für jeden Punkt der Geraden $A_{1} O_{1}$ die Gleichung

$$
r_{2} \cos \alpha_{2}+r_{3} \cos \alpha_{3}=0
$$

oder auch, wegen $\alpha_{1}=90^{\prime \prime}$,

$$
r_{1} \cos \alpha_{1}+r_{2} \cos \alpha_{2}+r_{3} \cos \alpha_{3}=0 \text {. }
$$

Bestimmt man ebenso den Minimumpunkt $O_{2}$ der Geraden $a_{2}$, so dass also $r_{1}: r_{3}=a_{1}: a_{3}$ oder $O_{2} A_{3}: O_{2} A_{1}=a_{1}^{2}: a_{3}^{2}$ ist, so gilt in Bezug auf $a_{2}$ für jeden Punkt der Geraden $\mathrm{A}_{2} \mathrm{O}_{2}$ die Gleichung

$$
r_{1} \cos \alpha_{1}+r_{2} \cos \alpha_{2}+r_{3} \cos \alpha_{3}=0 \text {. }
$$

Für den Durchschnittspunkt von $A_{1} O_{1}$ mit $A_{2} O_{2}$ gilt daher diese Gleichung in Bezug auf zwei Gerade, mithin in Bezug auf jede beliebige der Ebene; er ist also der gesuchte Minimumpunkt $O$.

Da Gleiches von $\mathrm{A}_{3} \mathrm{O}_{3}$ gilt, so bestimmt sich der Minimumpunkt $O$ eines Dreiecks als der gemeinschaftliche Durchschnittspunkt dreier Geraden, jede von einer Ecke des Dreiecks nach dem Minimumpunkt der gegenüberliegenden Seite. Die Linien $A_{1} O_{1}, A_{2} O_{2}, A_{3} O_{3}$, die den Schwerlinien analog sind, wollen wir Minimumaxen nennen.

2. Sind vier Gerade gegeben, welche sich in den Punkten $A, B, C$, $D, E, F$ schneiden, wo $r_{1}$ auf $A B, r_{2}$ auf $B C, r_{3}$ auf $C D, r_{4}$ auf $D A$ senkrecht steht, so ist der Punkt $O$ so zu bestimmen, dass in Bezug auf wenigstens zwei Gerade der Ebene die Bedingung

$$
r_{1} \dot{\cos } \alpha_{1}+r_{2} \cos \alpha_{2}+r_{3} \cos \alpha_{3}+r_{4} \cos \alpha_{4}=0
$$

erfällt ist. 
Wir suchen dieselbe zuerst in Bezug auf eine der Geraden, etwa $a_{4}$, zu erfüllen. Zu dem Zwecke construire man die durch $B, C, E$ gehenden Minimumaxen der über $a_{4}$ stehenden Dreiecke $A B F, D C F, A E D$ und nenne sie Axen erster Ordnung. Die erste, die Axe der Geraden $a_{1}$ und $a_{2}$, schneide $a_{3}$ in $M_{3}$; die zweite, die Axe von $a_{2}$ und $a_{3}$, schneide $a_{1}$ in $M_{1}$; die dritte, die Axe von $a_{3}$ und $a_{1}$, schneide $a_{2}$ in $M_{2}$. Alsdann ist, stets in Bezug auf $a_{4}$, für jeden Punkt der ersten Axe

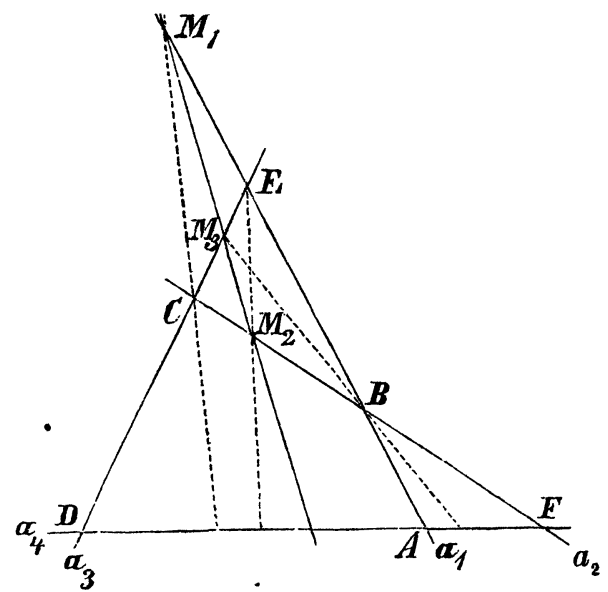
$r_{1} \cos \alpha_{1}+r_{2} \cos \alpha_{2}=0$, daher für $M_{3}$, da $r_{3}=0$ ist, $r_{1} \cos \alpha_{1}+r_{2} \cos \alpha_{2}+r_{3} \cos \alpha_{3}=0$. Für jeden Punkt der zweiten Axe ist $r_{2} \cos \alpha_{2}+r_{3} \cos \alpha_{3}=0$, daher für $M_{1}$ ebenfalls $r_{1} \cos \alpha_{1}+r_{2} \cos \alpha_{2}+r_{3} \cos \alpha_{3}=0$; und Gleiches gilt von $M_{2}$. Mithin liegen nach $\$$. 3, (2.) die Punkte $M_{1}, M_{2}, M_{3}$ auf einer Geraden von der Eigenschaft, dass für jeden ihrer Punkte $r_{1} \cos \alpha_{1}+r_{2} \cos \alpha_{2}+r_{3} \cos \alpha_{3}=0$, aber auch, wegen $\alpha_{4}=90^{\prime \prime}, r_{1} \cos \alpha_{1}+r_{2} \cos \alpha_{2}+r_{3} \cos \alpha_{3}+r_{4} \cos \alpha_{4}=0$ ist. Folglich ist die Gerade $M_{1} M_{2} M_{3}$ die zu $a_{4}$ gehörige Minimumaxe des Vierseits; sie enthält den Punkt $O$ und schneidet $a_{4}$ in dem Punkte $O_{4}$ dieser Geraden, für welchen die Quadratsumme der Abstände von den drei übrigen Geraden ein Minimum ist.

Indem man auf gleiche Weise die drei übrigen Minimumaxen construirt, bestimmt sich $O$ als der gemeinschaftliche Durchschnittspunkt von vier Axen, deren jede die zugehörige Gerade im Minimumpunkt derselben schneidet. Wir nennen sie Axen zweiter Ordnung.

Bezeichnet man mit $(2,3)_{1}$ die Axe erster Ordnung von $a_{2}, a_{3}$ in Bezug auf $a_{1}$, also die Gerade, für welche $r_{2} \cos \alpha_{2}+r_{3} \cos \alpha_{3}=0$ ist, ebenso mit $(2,3,4)_{1}$ die Gerade, für welche, in Bezug auf $a_{1}, r_{2} \cos \alpha_{2}+r_{3} \cos \alpha_{3}+r_{4} \cos \alpha_{4}=0$ ist, ferner mit $(2,3)_{1} 4$ den Durchschnittspunkt der Axe $(2,3)_{1}$ mit der Geraden $a_{4}$, endlich mit $(1,2,3,4)$ den Punkt $O$, so kann man dessen Construction durch folgendes Schema darstellen:

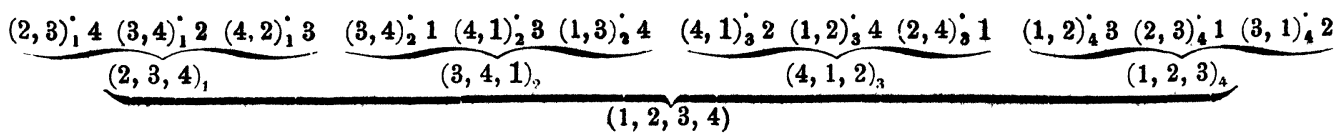

Die ganze Construction umfasst also $\frac{4.3}{1.2}=12$ Axen erster Ordnung, näm- 
lich jede Combination $(1,2)$ mit zwei Indices, 3 und 4 ; jede derselben giebt einen Durchschnittspunkt, nämlich $(1,2)_{3}$ mit $a_{4}$, wodurch man also zwölf Durchschnittspunkte erhält. Axen zweiter Ordnung $(1,2,3)_{4}$ giebt es vier, nämlich jede Combination $(1,2,3)$ mit einem Index; jede ist durch drei der zwölf Durchschnittspunkte bestimmt. Je drei Axen erster Ordnung schneiden sich in einem Punkte, wie $(1,2)_{3},(2,3)_{1},(3,1)_{2}$, und geben daher vier Durchschnittspunkte, durch deren jeden auch eine Axe zweiter Ordnung geht, nämlich $(1,2,3)_{4}$ durch $(1,2,3)$. Nimmt man also noch die sechs Punkte $A, B, C, \ldots$ hinzu, in deren jedem sich zwei gegebene Gerade (Axen $0^{\text {ter }}$ Ordnung) mit zwei Axen erster Ordnung schneiden, so hat man ein System von zwanzig Geraden mit eilf Punkten, in deren jedem sich je vier Gerade schneiden.

3. Sind fünf Gerade gegeben, so bestimmt sich der Minimumpunkt $O$ als der gemeinschaftliche Durchschnittspunkt von fünf Axen dritter Ordnung, nämlich der Axen $(2,3,4,5)_{1},(3,4,5,1)_{2},(4,5,1,2)_{3},(5,1,2,3)_{4},(1,2,3,4)_{5}$. Jede derselben ist durch vier Punkte bestimmt, und ihre Construction, z. B. die der ersten, ist durch folgendes Schema gegeben:

\begin{tabular}{|c|c|c|c|}
\hline$(2,3)_{1}^{\circ} 4(3,4)_{1}^{\circ} 2(4,2)_{1}^{\circ} 3$ & $(3,4) ; 5(4,5) ; 3(5,3) ; 4$ & $(4,5), 2(5,2), 4(2,4), 5$ & $(2,3), 5(3,5)$ \\
\hline$(2,3,4) ; 5$ & $(3,4,5) ; 2$ & $(4,5,2)_{1} 3$ & $(5,2,3)_{1}^{\circ} 4$ \\
\hline
\end{tabular}

Indem man auf gleiche Weise die vier übrigen Axen dritter Ordnung construirt, ergiebt sich Folgendes:

Man erhält $\frac{5(5-1)(5-2)}{1.2}=30$ Axen erster Ordnung, nämlich jede Combination $(1,2)$ mit $5-2=3$ Indices. Jede liefert $5-3=2$ Durchschnittspunkte, nämlich $(1,2)_{3}$ mit $a_{4}$ und $a_{5}$, so dass sich $\frac{5(5-1)(5-2)(5-3)}{1.2}=60$ Durchschnittspunkte ergeben. Axen zweiter 0rdnung giebt es $\frac{5(5-1)(5-2)(5-3)}{1.2 .3}=20$, nämlich jede Combination $(1,2,3)$ mit zwei Indices, jede Axe durch drei der sechzig Punkte bestimmt. Jede liefert einen Durchschnittspunkt, nämlich $(1,2,3)_{4}$ mit $a_{5}$, so dass solcher Durchschnittspunkte zwanzig entstehen. Axen dritter Ordnung giebt es $\frac{5(5-1)(5-2)(5-3)(5-4)}{1.2 .3 .4}=5$, nämlich jede Combination $(1,2,3,4)$ mit einem Index, jede durch vier der zwanzig Punkte bestimmt. Je drei Axen erster Ordnung schneiden sich in einem Punkte, nämlich $(1,2)_{3},(2,3)_{1},(3,1)_{2}$, und solcher Punkte giebt es zehn. Durch 
jeden derselben gehen noch zwei Axen zweiter Ordnung, nämlich $(1,2,3)_{4}$ und $(1,2,3)_{5}$. Ferner schneiden sich je vier Axen zweiter Ordnung in einem Punkte, nämlich $(1,2,3)_{+},(2,3,4)_{1},(3,4,1)_{2},(4,1,2)_{3}$, und solcher Punkte giebt es fünf. Durch jeden derselben geht auch eine Axe dritter Ordnung, nämlich $(1,2,3,4)_{5}$. Nimmt man dazu $O$ und die gegebenen fünf Geraden mit ihren zehn Durchschnittspunkten, in deren jedem sich noch drei Axen erster Ordnung treffen, so hat man ein System von sechzig Geraden mit sechsundzwanzig Punkten, in deren jedem sich fünf der Geraden schneiden.

4. Sind allgemein $k$ Gerade in der Ebene gegeben, so bestimmt sich ihr Minimumpunkt als der gemeinschaftliche Durchschnitt von $k$ Axen $k-2^{\text {ter }}$ Ordnung, deren jede die zugehörige Gerade in dem Minimumpunkt der letzteren schneidet; z. B. $(2,3,4, \ldots k)_{1}$ schneidet $a_{1}$ in $O_{1},(1,3,4, \ldots k)_{2}$ schneidet $a_{2}$ in $O_{2}$ u. s. w. Die ganze Construction umfasst $\frac{k(k-1)(k-2)}{2}$ Axen erster Ordnung, deren jede sich mit $k-3$ Geraden schneidet, wie $(1,2)_{3}$ mit $4,5, \ldots k$, wodurch $\frac{k(k-1)(k-2)(k-3)}{1.2}$ Durchschnittspunkte entstehen. Je drei dieser Punkte bestimmen eine Axe zweiter Ordnung, deren Anzahl $\frac{k(k-1)(k-2)(k-3)}{1.2 .3}$ beträgt. Jede dieser Axen schneidet $k-4$ Gerade, wie $(1,2,3)_{4}$ die Geraden $5,6, \ldots k$, wodurch $\frac{k(k-1)(k-2)(k-3)(k-4)}{1.2 .3}$ Durchschnittspunkte entstehen, von denen je vier eine Axe dritter Ordnung bestimmen, so dass die Anzahl der letzteren $\frac{k(k-1)(k-2)(k-3)(k-4)}{1.2 .3 .4}$ heträgt, u. s. w.

Je drei Axen erster Ordnung, wie $(1,2)_{3},(2,3)_{1},(3,1)_{2}$ schneiden sich in einem Punkte, dem Minimumpunkte von $a_{1}, a_{2}, a_{3}$, und solcher Punkte giebt es $\frac{k(k-1)(k-2)}{1.2 .3}$. Durch jeden derselben gehen aber auch $k-3$ Axen zweiter Ordnung, wie durch den oben genannten die Axen $(1,2,3)_{4},(1,2,3)_{5}, \ldots$ $(1,2,3)_{k}$. Je vier Axen zweiter Ordnung schneiden sich in einem Punkte, wie $(1,2,3)_{4},(2,3,4)_{1},(3,4,1)_{2},(4,1,2)_{3}$ im Minimumpunkte von $a_{1}, a_{2}, a_{3}, a_{4}$, und solcher Punkte giebt es $\frac{k(k-1)(k-2)(k-3)}{1.2 .3 .4}$. Durch jeden derselben gehen $k-4$ Axen dritter Ordnung, wie durch den genannten die Axen $(1,2,3,4)_{5},(1,2,3,4)_{6}, \ldots(1,2,3,4)_{k} ;$ u. s. w. Je $k-1$ Axen $k-3^{\text {ter }}$ Ordnung schneiden sich in einem Punkte; die Anzahl dieser Punkte ist $\frac{k(k-1)(k-2) \ldots 2}{1.2 \ldots(k-1)}=k$, und durch jeden derselben geht noch eine Axe $k-2^{\text {ter }}$ 
Ordnung. Endlich die $k$ Axen $k-2^{\text {ter }}$ Ordnung schneiden sich in einem Punkte, dem Minimumpunkte $O$ der gegebenen $k$ Geraden. Da die Summe der Binomialcoefficienten $\operatorname{der} k^{\text {ten }}$ Potenz $2^{k}$ ist, so erhält man, wenn man die gegebenen k Geraden und ihre $\frac{k(k-1)}{1.2}$ Durchschnittspunkte hinzunimmt, ein System von $k\left(2^{k-1}-k+1\right)$ Geraden mit $2^{k}-k-1$ Punkten, in deren jedem sich $k$ Gerade schneiden.

5. Sind zwei der gegebenen Geraden, $a_{1}$ und $a_{2}$, parallel, so vermindert sich die Anzahl der Axen, und insbesondere fallen die Minimumaxen $k-2^{\text {ter }}$ Ordnung der beiden parallelen Geraden zusammen. Die $k-2$ Axen erster Ordnung $(1,2)_{3},(1,2)_{4}, \ldots(1,2)_{k}$ fallen in eine mit $a_{1}$ parallele Gerade in gleichem senkrechten Abstande von $a_{1}$ und $a_{2}$ zusammen, und je zwei Axen erster Ordnung mit einer der gegebenen Geraden, indem

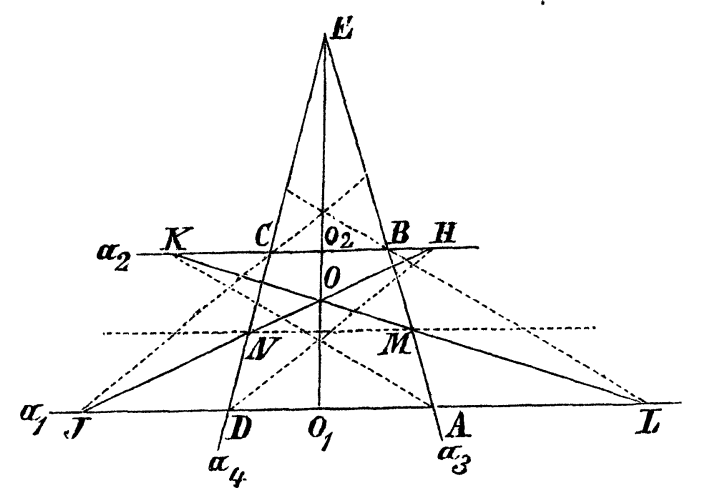
$(1, m)_{2}=(2, m)_{1}=a_{m}$ ist. Allgemein ist dann $(1,3,4, \ldots)_{2}=(2,3,4, \ldots)_{1}=$ $(3,4, \ldots)_{2}=(3,4, \ldots)_{1}$. Z. B. für das Paralleltrapez $A B C D$ hat man $(1,2)_{3}=$ $(1,2)_{4}=M N$, wo $M$ und $N$ die Mitten von $A B$ und $C D$ sind. Ferner ist

$$
\begin{array}{r}
(3,4,1)_{2}=(2,3,4)_{1}=(3,4)_{1} \\
=(3,4)_{2}=E O_{2} O_{1} .
\end{array}
$$

Die übrigen nach dem oben aufgestellten Schema zu bestimmenden Punkte sind:
$(4,1)_{3} \mathbf{2}=H$,
$(1,2)_{3} 4=N$,
$(2,4)_{3} 1=J$, so dass
$(4,1,2)_{3}=H N J$
$(1,2)_{4} 3=M$,
$(2,3)_{4} 1=L$,
$(3,1)_{4}^{:} 2=K$, so dass
$(1,2,3)_{4}=K M L$.

\$. 5.

Auf ganz analoge Weise ist die Construction des Punktes $O$ bei gegebenen Ebenen im Raume auszuführen.

1. Sind vier Ebenen, $f_{1}, f_{2}, f_{3}, f_{4}$ gegeben, welche eine dreiseitige Pyramide $A_{1} A_{2} A_{3} A_{4}$ bilden, deren Seitenflächen zugleich mit $f_{1}, f_{2}, f_{3}, f_{4}$ bezeichnet werden sollen, so dass $f_{1} \operatorname{dem}$ Punkte $A_{1}$ etc. gegenüberliegt, so muss in Bezug auf jede durch $O$ gelegte Gerade, mithin wenigstens in Bezug auf drei derselben die Bedingung

erfüllt sein.

$$
r_{1} \cos \alpha_{1}+r_{2} \cos \alpha_{2}+r_{3} \cos \alpha_{3}+r_{4} \cos \alpha_{4}=0
$$


Um zunächst den Minimumpunkt $o_{4}$ der Ebene $f_{4}$ d. h. den Púnkt zu finden, welcher in Bezug auf jede Gerade der Ebene $f_{4}$ der Bedingung $r_{1} \cos \alpha_{1}+r_{2} \cos \alpha_{2}+r_{3} \cos \alpha_{3}=0$ genügt, bestimme man den Punkt $M_{2}$ auf $A_{1} A_{3}$, für welchen, in Bezug auf $A_{1} A_{3}, r_{1} \cos \alpha_{1}+r_{3} \cos \alpha_{3}=0$, oder $r_{1} \cos \alpha_{1}=r_{3} \cos \left(180^{\prime \prime}-\alpha_{3}\right)$ ist. Dies ist leicht durch die Bemerkung, dass die Pyramiden $M_{2} A_{4} A_{2} A_{1}$ und und $M_{2} A_{4} A_{2} A_{3}$ die gemeinschaftliche Grundfläche $M_{2} A_{4} A_{2}$ haben, ihr Verhältniss also gleich dem ihrer Höhen oder gleich dem der Abschnitte $M_{2} A_{1}$ und $M_{2} A_{3}$ ist. Man hat demnach

folglich

$$
f_{1} r_{1}: f_{3} r_{3}=M_{2} A_{3}: M_{2} A_{1}=\frac{r_{1}}{\cos \alpha_{1}}: \frac{r_{3}}{\cos \left(180^{\circ}-\alpha_{3}\right)}=r_{1}^{2}: r_{3}^{2},
$$

$$
\begin{gathered}
r_{1}: r_{3}=f_{1}: f_{3}, \\
M_{2} A_{3}: M_{2} A_{1}=f_{1}^{2}: f_{3}^{2} .
\end{gathered}
$$

Es ist also die Pyramidenkante $A_{1} A_{3}$ in zwei Abschnitte zu theilen, die sich wie die Quadrate der anstossenden Pyramidenflächen verhalten, und es sind dann die Abstände des Theilpunktes $M_{2}$ von den Seitenflächen denselben proportional.

Nun ist aber für jeden Punkt dèr Geraden $A_{2} M_{2}$ in Bezug auf $A_{1} A_{3}$

$$
r_{1} \cos \alpha_{1}+r_{3} \cos \alpha_{3}=0
$$

daher auch, wegen $\alpha_{2}=90^{\circ}$,

$$
r_{1} \cos \alpha_{1}+r_{2} \cos \alpha_{2}+r_{3} \cos \alpha_{3}=0 \text {. }
$$

Theilt man daher in gleicher Weise $A_{2} A_{3}$ in $M_{1}$ so, dass $M_{1} A_{3}: M_{1} A_{2}=f_{2}^{2}: f_{3}^{2}$, und $A_{2} A_{1}$ in $M_{3}$ so, dass $M_{3} A_{2}: M_{3} A_{1}=f_{1}^{2}: f_{2}^{2}$, so ergiebt sich $O_{4}$ als der gemeinschaftliche Durchschnittspunkt der drei Geraden $A_{1} M_{1}, A_{2} M_{2}, A_{3} M_{3}$, und es gilt für denselben

$$
r_{1}: r_{2}: r_{3}=f_{1}: f_{2}: f_{3} .
$$

Ferner ist $O_{4}$ der Scheitel dreier Dreiecke, deren Verhältniss ist

indem z. B.

$$
A_{3} O_{4} A_{2}: A_{2} O_{4} A_{1}: A_{1} O_{4} A_{3}=f_{1}^{2}: f_{3}^{2}: f_{2}^{2} \text {, }
$$

$$
O_{4} A_{2} A_{1}: O_{4} A_{2} A_{3}=M_{2} A_{1}: M_{2} A_{3}=f_{3}^{2}: f_{1}^{2} \text {. }
$$

Da nun aber für $A_{4}, r_{1}=r_{2}=r_{3}=0$, also $r_{1} \cos \alpha_{1}+r_{2} \cos \alpha_{2}+r_{3} \cos \alpha_{3}=0$ ist, so ist nach \$. 3, 3. für jeden Punkt der Geraden $A_{4} O_{4}$ in Bezug auf jede Gerade der Ebene $f_{4}$

$$
r_{1} \cos \alpha_{1}+r_{2} \cos \alpha_{2}+r_{3} \cos \alpha_{3}=0
$$

folglich auch, wegen $\alpha_{4}=90^{\prime \prime}$,

$$
r_{1} \cos \alpha_{1}+r_{2} \cos \alpha_{2}+r_{3} \cos \alpha_{3}+r_{4} \cos \alpha_{4}=0 ;
$$


daher ist $A_{4} O_{4}$ die zu $f_{4}$ gehörige Minimumaxe der Pyramide und wird als Axe erster Ordnung durch $(1,2,3)_{4}$ bezeichnet.

Theilt man in gleicher Weise die übrigen drei Kanten der Pyramide, so erhält man auf $f_{1}, f_{2}, f_{3}$ die Punkte $O_{1}, O_{2}, O_{3}$, und $O$ selbst als den gemeinschaftlichen Durchschnittspunkt der vier Axen $A_{1} O_{1}, A_{2} O_{2}, A_{3} O_{3}, A_{4} O_{4}$. Der Minimumpunkt einer dreiseitigen Pyramide bestimmt sich also als der gemeinschaftliche Durchschnittspunkt von vier Geraden, jede von einer Ecke nach dem Minimumpunkte der gegenüberliegenden Seitenfläche gehend. Seine Abstände von den Seitenflächen sind diesen proportional, indem z. B. für jeden Punkt von $A_{4} O_{4}, r_{1}: r_{2}: r_{3}=f_{1}: f_{2}: f_{3}$, für jeden Punkt von $A_{3} O_{3}, r_{1}: r_{2}: r_{4}=f_{1}: f_{2}: f_{4}$, mithin für den Durchschnittspunkt beider $r_{1}: r_{2}: r_{3}: r_{4}=f_{1}: f_{2}: f_{3}: f_{4}$ ist.

2. Dieses Verfahren zur Construction des Punktes $O$ lässt sich in gleicher Weise auf beliebig viele Ebenen ausdehnen, wie in der Ebene auf beliebig viele Gerade: Der Minimumpunkt von $k$ Ebenen bestimmt sich als der gemeinschaftliche Durchschnittspunkt von $k$ Axen, deren jede die zugehörige Ebene im Minimumpunkte derselben schneidet. Es wird, nach dem Vorhergehenden, das folgende Schema für fünf Ebenen ohne Weiteres verständlich sein.

\begin{tabular}{|c|c|c|c|c|}
\hline$(1,2,3)_{5} 4$ & $(2,3,4)_{1} 5$ & $(3,4,5)_{2} 1$ & $(4,5,1)_{3} 2$ & $(5,1,2)_{4} 3$ \\
\hline$(2,3,4)_{5} 1$ & $(3,4,5)_{1}^{\prime} 2$ & $(4,5,1)_{2} 3$ & $(5,1,2)_{3} 4$ & $(1,2,3)_{4} 5$ \\
\hline$(3,4,1)_{5}^{:} 2$ & $(4,5,2)_{1}^{;} 3$ & $(5,1,3)_{2} 4$ & $(1,2,4)_{3} 5$ & $(2,3,5)_{4}^{;} 1$ \\
\hline$(4,1,2)_{5} 3$ & $(5,2,3)_{1} 4$ & $(1,3,4)_{2} 5$ & $(2,4,5)_{3}^{\circ} 1$ & $(3,5,1)_{4} 2$ \\
\hline$(1,2,3,4)_{5}$ & $(2,3,4,5)_{1}$ & $(3,4,5,1)_{2}$ & $(4,5,1,2)_{3}$ & $(5,1,2,3)_{4}$ \\
\hline
\end{tabular}

$(1,2,3,4,5)$

Jede Axe zweiter Ordnung wird demnach durch vier Punkte bestimmt, die nach $\$$. 3, 3. auf einer Geraden liegen.

Wir übergehen die Betrachtungen, die sich über die Anzahl der Axen und ihrer Durchschnittspunkte anknüpfen lassen.

3. Wenn zwei der Ebenen, $f_{1}$ und $f_{2}$, parallel sind, so vermindert sich die Anzahl der Axen, und insbesondere fallen die Minimumaxen von $f_{1}$ und $f_{2}$ mit zwei Axen nächst niederer Ordnung zusammen, indem allgemein $(2,3,4, \ldots)_{1}=(1,3,4, \ldots)_{2}=(3,4, \ldots)_{1}=(3,4, \ldots)_{2}$ ist. Ist von vier Ebenen $f_{1} \| f_{2}$, und schneiden sich $f_{3}$ und $f_{4}$ in der Geraden $A_{1} A_{2}$, wo $A_{1}$ in $f_{1}$ und $A_{2}$ in $f_{2}$ liegt, so ist $(2,3,4)_{1}=(3,4,1)_{2}=(3,4)_{1}=A_{1} A_{2}$; ferner ist $(1,2,3)_{4}$ die Durchschnittsgerade von $f_{3}$ mit der zu $f_{1}$ und $f_{2}$ parallelen Ebene, welche 
den Abstand beider halbirt; $(4,1,2)_{3}$ ist die Durchschnittsgerade von $f_{4}$ mit derselben Ebene, und $O$ ist die Mitte von $A_{1} A_{2}$.

Wenden wir dies auf eine abgestumpfte dreiseitige Pyramide an mit den parallelen Ebenen $A_{4} A_{5} A_{3}=f_{2}$ und $B_{4} B_{5} B_{3}=f_{1}$, während $A_{4} A_{5} B_{5} B_{7}$ mit $f_{3}$ u. s. w. bezeichnet wird, so ist $(2,3,4,5)_{1}=(3,4,5,1)_{2}=(3,4,5)_{1}$ $=(3,4,5)_{2}=A_{1} O_{1} O_{2}$. Sei ferner $(2,4,5)_{3}=A_{3} O_{3},(4,5,1)_{3}=B_{3} O_{3}^{\prime}$, wo $B_{3} O_{3}^{\prime} \| A_{3} O_{3}$ ist, und $(5,1,2)_{3} 4=(1,2,4)_{3} 5=M_{3}$, d. i. die Mitte von $A_{3} B_{3}$, so hat man $(4,5,1,2)_{3}$ bestimmt durch die drei Punkte $A_{3} O_{3}^{\prime} f_{1}, M_{3}, B_{3} O_{3}^{\prime} f_{2}$. Bei analoger Bezeichnung wird ferner $(5,1,2,3)_{4}$ durch die Punkte $A_{4} O_{4} f_{1}$, $M_{4}, B_{4} O_{4}^{\prime} f_{2}$, und endlich $(1,2,3,4)_{5}$ durch die drei Punkte $A_{5} O_{5}^{\prime} f_{1}, M_{5}$, $B_{5} O_{5}^{\prime \cdot} f_{2}$ bestimmt.

\section{\$. 6 .}

1. Setzt man in der $\$$. 2,1 . gefundenen Gleichung für die Quadratsumme der senkrechten Abstände $\Sigma r^{\prime 2}$ aus einem beliebigen Punkte $O^{\prime}$ die Zunahme $\Sigma r^{\prime 2}-\Sigma r^{2}=c^{2}$, so hat man

$$
c^{2}=t^{2} \Sigma \cos ^{2} r t
$$

Daher wächst diese Zunahme auf einer durch $O$ gelegten Geraden proportional dem Quadrate des Abstandes vom Minimumpunkte.

Um den Ort der Punkte $O^{\prime}$ constanter Quadratsumme der Abstände zu finden, lege man bei gegebenen Geraden in ihrer Ebene durch $O$ eine beliebige Nullinie, mit welcher $t$ den Winkel $\varphi$, und $r$ den Winkel $\alpha$ bildet. Alsdann ist $r t=\alpha-\varphi$, und man hat daher, wenn $c^{2}$ constant sein soll, zwischen $t$ und $\varphi$ die Relation

$$
t^{2}=\frac{c^{2}}{\cos ^{2} \psi \Sigma \cos ^{2} \alpha+\sin ^{2} \varphi \Sigma \sin ^{2} \alpha+2 \sin \varphi \cos \varphi \Sigma \sin \alpha \cos \alpha} .
$$

Die Punkte constanter Quadratsumme der Abstände von gegebenen Geraden liegen daher auf einer Ellipse, deren Mittelpunkt der Minimumpunkt ist.

Nimmt man als Nullinie eine der beiden Axen der Ellipse, so muss für jede derselben $\Sigma \sin 2 \alpha=0$ sein, und das Verschwinden dieser Summe wird durch eine Drehung der bisherigen Nulllinie um einen Winkel $u$ erreicht, der durch die Gleichung

$$
\tan 22 u=\frac{\Sigma \sin 2 \alpha}{\Sigma \cos 2 \alpha}
$$

bestimmt ist. Werden daher die Winkel $\alpha$ gegen die Axe $2 a$ der Ellipse gerechnet, so ist 


$$
t^{2}=\frac{c^{2}}{\cos ^{2} \varphi \sum \cos ^{2} \alpha+\sin ^{2} \varphi \sum \sin ^{2} \alpha} .
$$

Die Quadrate der Halbaxen $a$ und $b$ haben die Grössen

$$
a^{2}=\frac{c^{2}}{\Sigma \cos ^{2} \alpha}, \quad b^{2}=\frac{c^{2}}{\Sigma \sin ^{2} \alpha},
$$

woraus folgt, wenn $k$ die Anzahl der Geraden ist,

$$
\frac{1}{a^{2}}+\frac{1}{b^{2}}=\frac{k}{c^{2}} \text {. }
$$

Von der Schaar concentrischer und ähnlicher Ellipsen, die daher zu verschiedenen Werthen von $c^{2}$ gehören, berührt je eine eine Gerade $a_{m}$ in dem Minimumpunkte $O_{m}$ dieser Geraden; denn zu jedem anderen Punkte von $a_{m}$ gehört eine Ellipse von grösseren Axen und daher ein grösseres $c^{2}$. Daraus folgt aber, dass $O O_{m}$ der zu $a_{m}$ (d. i. zu dem mit $a_{m}$ parallelen Durchmesser) conjugirte Durchmesser der Ellipse ist. Mithin ist jede der $k$ Minimumaxen von $k$ gegebenen Geraden der zur zugehörigen Geraden conjugirte Durchmesser der Ellipsen constanter Quadratsumme der Abstände.

Die Ellipsen gehen in Kreise über, wenn $\Sigma \cos ^{2} \alpha=\Sigma \sin ^{2} \alpha$ ist, also in Bezug auf jede Gerade der Ebene $\Sigma \cos ^{2} \alpha$ denselben Werth hat $\left.{ }^{*}\right)$. Alsdann stehen die Axen $k-2^{\text {ter }}$ Ordnung auf ihren Geraden senkrecht, fallen also mit den Abständen $r$ zusammen.

2. Ist $O$ der Minimumpunkt einer Ebene in Bezug auf andere Ebenen, sind $x$ und $y$ zwei in dieser Ebene durch $O$ gelegte rechtwinklige Axen, und bezeichnet man die Winkel $r x, r y, t x$ mit $\alpha, \beta, \varphi$, so ist

$$
\begin{gathered}
\cos r t=\cos \varphi \cdot \cos \alpha+\sin \varphi \cdot \cos \beta \\
t^{2}=\frac{c^{2}}{\cos ^{2} \varphi \Sigma \cos ^{2} \alpha+\sin ^{2} \varphi \Sigma \cos ^{2} \beta+2 \sin \varphi \cos \varphi \Sigma \cos \alpha \cos \beta} .
\end{gathered}
$$

Mithin liegen die Punkte einer Ebene, für welche die Quadratsumme der Abstände von gegebenen Ebenen constant ist, auf einer Ellipse, deren Mittelpunkt der Minimumpunkt dieser Ebene ist. Nimmt man als Coordinatenaxen die Axen der Ellipse, so muss in Bezug auf dieselben $\Sigma \cos \alpha \cos \beta=0$ sein, daher

$$
t^{2}=\frac{c^{2}}{\cos ^{2} \varphi \Sigma \cos ^{2} \alpha+\sin ^{2} \varphi \Sigma \sin ^{2} \alpha} .
$$

Jede Durchschnittsgerade der Ebene mit den übrigen Ebenen wird von einer der, verschiedenen Werthen von $c^{2}$ entsprechenden, concentrischen und ähnlichen

*) S. §. 14, 1. und 3 . 
Ellipsen in dem Punkte dieser Geraden berührt, fur welchen die Quadratsumme der Abstände von den gegebenen Ebenen ein Minimum ist. Die Ellipsen gehen in Kreise über, wenn $\Sigma \cos ^{2} \alpha=\Sigma \cos ^{2} \beta$ und daher $\Sigma \cos ^{2} \alpha$ in Bezug auf jede Gerade der Ebene constant ist.

3. Ist $O$ der Minimumpunkt von $k$ Ebenen, und sind $\alpha, \beta, \gamma$ die Winkel, die $r$ mit drei beliebig durch $O$ gelegten rechtwinkligen Axen bildet, $\xi, \eta, \zeta$ die Winkel von $t$ mit denselben Axen, so folgt

$$
\cos r t=\cos \alpha \cdot \cos \xi+\cos \beta \cdot \cos \eta+\cos \gamma \cdot \cos \zeta
$$

Setzt $\cdot$ man diesen Werth in

$$
t^{2}=\frac{c^{2}}{\Sigma \cos ^{2} r t}
$$

ein, so erhält man für $\Sigma \cos ^{2} r t$ den Ausdruck

$$
\begin{aligned}
\cos ^{2} \xi \Sigma \cos ^{2} \alpha+\cos ^{2} \eta \Sigma \cos ^{2} \beta+\cos ^{2} \zeta \Sigma \cos ^{2} \gamma+2 \cos \xi \cos \eta \Sigma \cos \alpha \cos \beta \\
+2 \cos \eta \cos \zeta \Sigma \cos \beta \cos \gamma+2 \cos \zeta \cos \xi \Sigma \cos \gamma \cos \alpha .
\end{aligned}
$$

Hieraus folgt, dass die Punkte constanter Quadratsumme der Abstände auf einem Ellipsoid liegen, dessen Mittelpunkt der Minimumpunkt ist.

Nimmt man die Axen dieses Ellipsoids als Coordinatenaxen an, so muss in Bezug auf dieselben $\Sigma \cos \alpha \cos \beta=\Sigma \cos \beta \cos \gamma=\Sigma \cos \gamma \cos \alpha=0$ sein, folglich

$$
t^{2}=\frac{c^{2}}{\cos ^{2} \xi \Sigma \cos ^{2} \alpha+\cos ^{2} \eta \Sigma \cos ^{2} \beta+\cos ^{2} \zeta \Sigma \cos ^{2} \gamma} .
$$

Sind $f, g, h$ die Halbaxen des Ellipsoids, so ist

$$
\begin{gathered}
f^{2}=\frac{c^{2}}{\Sigma \cos ^{2} \alpha}, \quad g^{2}=\frac{c^{2}}{\Sigma \cos ^{2} \beta}, \quad h^{2}=\frac{c^{2}}{\Sigma \cos ^{2} \gamma}, \\
\frac{1}{f^{2}}+\frac{1}{g^{2}}+\frac{1}{h^{2}}=\frac{k}{c^{2}} .
\end{gathered}
$$

Verschiedenen Werthen von $c^{2}$ entsprechen concentrische, ähnliche Ellipsoide, welche die gegebenen Ebenen in den oben erwähnten Ellipsen constanter Quadratsumme schneiden; jede Ebene wird von einem der Ellipsoide im Mittelpunkte dieser Ellipsen, der zugleich der Minimumpunkt dieser Ebene in Bezug auf die übrigen Ebenen ist, berührt. Jede Minimumaxe $O O_{m}$ (\$. 5) ist daher der zur zugehörigen Ebene $f_{m}$ (d. i. zur parallelen Diametralebene) conjugirte Durchmesser der Ellipsoide.

Die Ellipsoide gehen in Kugelflächen über, wenn

$$
\Sigma \cos ^{2} \alpha=\Sigma \cos ^{2} \beta=\Sigma \cos ^{2} \gamma,
$$

also $\Sigma \cos ^{2} \alpha$ in Bezug auf jede beliebige Gerade constant ist. 
4. Aus der Grundgleichung

$$
\Sigma r^{2}-\Sigma r^{2}=t^{2} \Sigma \cos ^{2} r t
$$

lässt sich noch eine bemerkenswerthe Folgerung ziehen. Wegen $r-r^{\prime}=t \cos r t$ kànn man nämlich dafür schreiben

$$
\begin{gathered}
\Sigma r^{\prime 2}-\Sigma r^{2}=\Sigma\left(r-r^{\prime}\right)^{2}, \\
\Sigma r^{2}=\Sigma r . r^{\prime} .
\end{gathered}
$$

Es ist also die Summe der Producte der Abstände eines beliebigen Punktes mit den zugehörigen des Minimumpunktes constant, nämlich gleich der Minimalsumme der Quadrate der Abstände. Sind z. B. die gegebenen Geraden einem Kreise oder die Ebenen einer Kugel vom Halbmesser $\omega$ umschrieben, so folgt

$$
\Sigma r^{2}=\varrho \Sigma r .
$$

Oder gehen die Geraden oder Ebenen mit Ausnahme der ersten durch einen und denselben Punkt im Abstande $h$ von dieser, so folgt

$$
\Sigma r^{2}=h \cdot r_{1}
$$

welche Relation z. B. auf jede Pyramide anwendbar ist.

\$. 7.

Im Folgenden sollen die Abstände $r$ des Minimumpunktes $O$ von den gegebenen Geraden oder Ebenen durch die Bestimmungsstücke derselben ausgedrückt werden.

In Bezug auf drei Gerade, welche auf einander die ihren Durchschnittspunkten $A_{1}, A_{2}, A_{3}$ gegenüberliegenden Abschnitte $a_{1}, a_{2}, a_{3}$ bilden, haben wir $\$ .4,1$. gesehen, dass die Abstände des Minimumpunktes den zugehörigen Dreiecksseiten proportional sind, also

$$
r_{1}: r_{2}: r_{3}=a_{1}: a_{2}: a_{3} \text {. }
$$

Nimmt man zu diesen Proportionen noch die Gleichung

$$
a_{1} r_{1}+a_{2} r_{2}+a_{3} r_{3}=2 f=a_{1} h_{1}=a_{2} h_{2}=a_{3} h_{3},
$$

worin $h_{1}, h_{2}, h_{3}$ die Höhen des Dreiecks und $f$ seine Fläche bezeichnet, so folgt

$$
\begin{aligned}
& r_{1}=h_{1} \frac{a_{1}^{2}}{a_{1}^{2}+a_{2}^{2}+a_{3}^{2}}=2 f \frac{a_{1}}{a_{1}^{2}+a_{2}^{2}+a_{3}^{2}}, \\
& r_{2}=h_{2} \frac{a_{2}^{2}}{a_{1}^{2}+a_{2}^{2}+a_{3}^{2}}=2 f \frac{a_{2}}{a_{1}^{2}+a_{2}^{2}+a_{3}^{2}}, \\
& r_{3}=h_{3} \frac{a_{3}^{2}}{a_{1}^{2}+a_{2}^{2}+a_{3}^{2}}=2 f \frac{a_{3}}{a_{1}^{2}+a_{2}^{2}+a_{3}^{2}},
\end{aligned}
$$


woraus sich als Minimalsumme ergiebt:

$$
r_{1}^{2}+r_{2}^{2}+r_{3}^{2}=\frac{4 f^{2}}{a_{1}^{2}+a_{2}^{2}+a_{3}^{2}} .
$$

Zieht man Gerade von $O$ nach $A_{1}, A_{2}, A_{3}$, so erhält man drei Dreiecke, die sich wie die Quadrate der Dreiecksseiten verhalten, auf denen sie stehen,

$$
A_{1} O A_{2}: A_{2} O A_{3}: A_{3} O A_{1}=a_{3}^{2}: a_{1}^{2}: a_{2}^{2},
$$

indem z. B. $A_{1} O A_{2}: A_{2} O A_{3}=a_{3} r_{3}: a_{1} r_{1}=a_{3}^{2}: a_{1}^{2}$.

Das Gleichungssystem, durch welches die $r$ bestimmt sind, kann man auch in folgender Gestalt schreiben:

$$
\begin{aligned}
-a_{3} r_{1}+a_{1} r_{3} & =0, \\
-a_{3} r_{2}+a_{2} r_{3} & =0, \\
a_{1} r_{1}+a_{2} r_{2}+a_{3} r_{3} & =2 f,
\end{aligned}
$$

so dass der gemeinschaftliche Nenner der $r$ durch die symmetrische Determinante dargestellt wird:

$$
\left|\begin{array}{rrr}
-a_{3} & 0 & a_{1} \\
0 & -a_{3} & a_{2} \\
a_{1} & a_{2} & a_{3}
\end{array}\right|=a_{3} . R
$$

wo $R=a_{1}^{2}+a_{2}^{2}+a_{3}^{2}$.

Als specieller Fall ist das rechtwinklige Dreieck erwähnenswerth. Sei $A_{3}$ ein rechter Winkel, so ist, wegen $O_{3} A_{1}: O_{3} A_{2}=a_{2}^{2}: a_{1}^{2}, A_{3} O_{3}$ die zur Hypotenuse gehörige Höhe $h$ und folglich $r_{3}=\frac{1}{2} h$; so dass $O$ die Mitte dieser Höhe ist. Die Ellipsen constanter Quadratsumme haben die eine Axe a parallel zur Hypotenuse $A_{1} A_{2}$, weil $A_{3} O_{3}$ senkrecht zu $A_{1} A_{2}$ und zu $A_{1} A_{2}$ conjugirt ist. Das Quadrat des Axenverhältnisses ist

$$
\frac{b^{2}}{a^{2}}=\frac{\Sigma \cos ^{2} \alpha}{\Sigma \sin ^{2} \alpha}=\frac{\sin ^{2} A_{2}+\sin ^{2} A_{1}}{1+\cos ^{2} A_{2}+\cos ^{2} A_{1}}=\frac{1}{2},
$$

also für alle rechtwinkligen Dreiecke constant. Die Minimalsumme der Quadrate der Abstände ist $r_{1}^{2}+r_{2}^{2}+r_{3}^{2}=O O_{3}^{2}+O A_{3}^{2}=\frac{1}{2} h^{2}$, also gleich dem halben Quadrate der Höhe. Die Zunahme $c^{2}$ der Quadratsumme der Abstände von $O$ bis zu den Punkten der Ellipse mit den Halbaxen $a$ und $b$ wird bestimmt durch

$$
\frac{1}{a^{2}}+\frac{1}{b^{2}}=\frac{3}{c^{2}}
$$

woraus wegen $a^{2}=2 b^{2}$ folgt $c^{2}=a^{2}$; diese Zunahme ist also gleich dem Quadrate der grossen Halbaxe. 
5. 8 .

1. Wenn vier Gerade gegeben sind, so können die Abstände $r$ im Allgemeinen auf sechs verschiedene Weisen ausgedrückt werden, nämlich jedesmal durch die Seiten und Flächen der beiden Dreiecke, die in jedem der sechs Durchschnittspunkte an einander stossen. Um nicht zu weitläufig zu werden, übergehen wir jetzt die aus diesen verschiedenen Ausdrucksweisen hervorgehenden Identitäten.

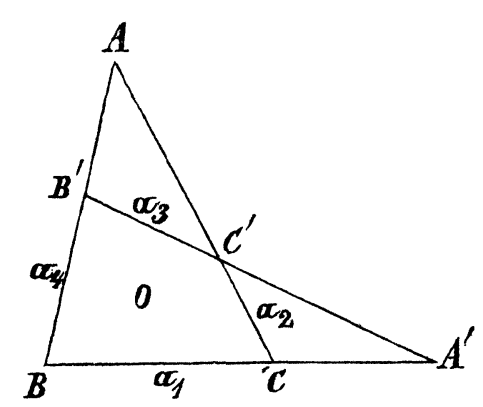

Sei in beistehender Figur $A B=c, A C=b$, $B C=a, A B^{\prime}=c^{\prime}, A C^{\prime}=b^{\prime}, B^{\prime} C^{\prime}=a^{\prime}$, und seien $r_{1}, r_{2}, r_{3}, r_{4}$ der Reihe nach die Abstände des Punktes $O$ von $B C, C A, B^{\prime} C^{\prime}, A B$. Da nun die Bedingungsgleichung $\Sigma r \cos \alpha=0$ gleichbedeutend mit $\Sigma r \sin \alpha=0$ ist und nur in Bezug auf zwei Gerade der Ebene erfüllt zu sein braucht, um für alle zu gelten, so wird ihr durch die beiden Gleichungen

$$
\begin{aligned}
& r_{3} \sin C^{\prime}+r_{4} \sin A-r_{1} \sin C=0, \\
& r_{3} \sin B^{\prime}+r_{2} \sin A-r_{1} \sin B=0
\end{aligned}
$$

Genüge geleistet, die man erhält, indem man einmal $r_{2}$ dann $r_{4}$ als Nulllinie nimmt. Dividirt man beide Gleichungen mit $\sin A$ und setzt statt der Sinus die ihnen proportionalen Dreiecksseiten, so folgt

$$
\text { (1.) } \quad\left\{\begin{array}{l}
a c^{\prime} r_{3}+a a^{\prime} r_{4}-c a^{\prime} r_{1}=0, \\
a b^{\prime} r_{3}+a a^{\prime} r_{2}-b a^{\prime} r_{1}=0 .
\end{array}\right.
$$

Dazu kommen, wenn $\triangle A B C=f$ und $\triangle A B^{\prime} C^{\prime}=f^{\prime}$ gesetzt wird, noch die Gleichungen

$$
\begin{aligned}
a r_{1}+b r_{2}+c r_{4} & =2 f, \\
-a^{\prime} r_{3}+b^{\prime} r_{2}+c^{\prime} r_{4} & =2 f^{\prime} .
\end{aligned}
$$

Man hat demnach zur Bestimmung der $r$ folgendes Gleichungssystem aufzuläsen:

$$
\begin{aligned}
a a^{\prime} r_{4}-c a^{\prime} r_{1}+a c^{\prime} r_{3} & =0, \\
+a a^{\prime} r_{2}-b a^{\prime} r_{1}+a b^{\prime} r_{3} & =0, \\
-c r_{4}-b r_{2}-a r_{1} & =-2 f, \\
+c^{\prime} r_{4}+b r_{2},-a^{\prime} r_{3} & =2 f^{\prime} .
\end{aligned}
$$

Der gemeinschaftliche Nenner der $r$ wird daher durch folgende symmetrische 
Determinante dargestellt:

$$
a a^{\prime}\left|\begin{array}{cccc}
a a^{\prime} & 0 & -c & +c^{\prime} \\
0 & a a^{\prime} & -b & +b^{\prime} \\
-c & -b & -\frac{a}{a^{\prime}} & 0 \\
c^{\prime} & b^{\prime} & 0 & -\frac{a^{\prime}}{a}
\end{array}\right|=a a^{\prime} R,
$$

wo sich für $R$ der Werth ergiebt:

$$
R=a^{2} a^{\prime 2}+a^{2}\left(b^{\prime 2}+c^{\prime 2}\right)+a^{\prime 2}\left(b^{2}+c^{2}\right)+\left(b c^{\prime}-b^{\prime} c\right)^{2} .
$$

Durch Auflösung des obigen Gleichungssystems erhält man ferner:

$$
\left\{\begin{array}{l}
\boldsymbol{R} . r_{1}=a\left[2 f\left(a^{\prime 2}+b^{\prime 2}+c^{\prime 2}\right)-2 f^{\prime}\left(b b^{\prime}+c c^{\prime}\right)\right], \\
\boldsymbol{R} . r_{3}=a^{\prime}\left[2 f\left(b b^{\prime}+c c^{\prime}\right)-2 f^{\prime}\left(a^{2}+b^{2}+c^{2}\right)\right], \\
\boldsymbol{R} . r_{2}=2 f\left[b\left(a^{\prime 2}+c^{\prime 2}\right)-b^{\prime} c c^{\prime}\right]+2 f^{\prime}\left[b^{\prime}\left(a^{2}+c^{2}\right)-b c^{\prime} c\right], \\
\boldsymbol{R} . r_{4}=2 f\left[c\left(a^{\prime 2}+b^{\prime 2}\right)-b b^{\prime} c^{\prime}\right]+2 f^{\prime}\left[c^{\prime}\left(a^{2}+b^{2}\right)-b^{\prime} b c\right] .
\end{array}\right.
$$

Setzt man für $2 f$ und $2 f^{\prime}$ die Werthe $2 f=b c \sin A$ und $2 f^{\prime}=b^{\prime} c^{\prime} \sin A$, so gehen diese Gleichungen in folgende über:

$$
\left\{\begin{array}{l}
\boldsymbol{R} . r_{1}=a \sin A\left[a^{\prime 2} b c+b^{\prime 2} b\left(c-c^{\prime}\right)+c^{\prime 2} c\left(b-b^{\prime}\right)\right] \\
\boldsymbol{R} . r_{3}=a^{\prime} \sin A\left[-a^{2} b^{\prime} c^{\prime}+b^{2} b^{\prime}\left(c-c^{\prime}\right)+c^{2} c^{\prime}\left(b-b^{\prime}\right)\right] \\
\boldsymbol{R} . r_{2}=\sin A\left[a^{2} b^{\prime 2} c^{\prime}+a^{\prime 2} b^{2} c+c c^{\prime}\left(b-b^{\prime}\right)\left(b c^{\prime}-b^{\prime} c\right)\right] \\
\boldsymbol{R} . r_{4}=\sin A\left[a^{2} b^{\prime} c^{\prime 2}+a^{\prime 2} b c^{2}+b b^{\prime}\left(c-c^{\prime}\right)\left(c b^{\prime}-c^{\prime} b\right)\right]
\end{array}\right.
$$

2. Wir ziehen zunächst den Fall in nähere Betrachtung, dass die Abstände $r$ den zugehörigen Vierecksseiten proportional sind. Dies ist auf zweierlei Weise möglich.

1.) Die $r$ sind den Seiten des Vierecks $B C C^{\prime} B^{\prime}$ proportional, also

$$
r_{1}: r_{2}: r_{3}: r_{4}=B C: C C^{\prime}: C^{\prime} B^{\prime}: B^{\prime} B=a: b-b^{\prime}: a^{\prime}: c-c^{\prime} .
$$

Es braucht nur eine von diesen Proportionen, etwa $r_{1}: r_{3}=a: a^{\prime}$, erfüllt $\mathrm{zu}$ sein, so ergeben sich die übrigen aus den Gleichungen (1.) von selbst, wie auch daraus folgt, dass dann das Viereck, dessen Seiten der Reihe nach mit $r_{1}, r_{2}, r_{3}, r_{4}$ gleich und gleich gerichtet sind, mit dem gegebenen ähnlich ist (vergl. die Schlussbemerkung \$. 2, 3.). Die Bedingung $r_{1}: r_{3}=a: a^{\prime}$ liefert aber aus den beiden ersten Gleichungen unter (4.) die Bedingungsgleichung

$$
a^{2} b^{\prime} c^{\prime}+a^{\prime 2} b c=\left(b-b^{\prime}\right)\left(c-c^{\prime}\right)\left(b b^{\prime}+c c^{\prime}\right)
$$


Nimmt man die allgemeine Relation

$$
a^{2} b^{\prime} c^{\prime}-a^{\prime 2} b c=b^{\prime} c^{\prime}\left(b^{2}+c^{2}\right)-b c\left(b^{\prime 2}+c^{\prime 2}\right)
$$

hinzu, welche sich aus $a^{2}=b^{2}+c^{2}-2 b c \cos A$ und $a^{\prime 2}=b^{\prime 2}+c^{\prime 2}-2 b^{\prime} c^{\prime} \cos A$ durch Elimination von $\cos A$ ergiebt, so kann man, indem man diese Gleichungen addirt und subtrahirt, $a^{2}$ und $a^{\prime 2}$ durch vier Bestandtheile des Vierecks, nämlich $b, b^{\prime}, c, c^{\prime}$, also die zwischen $a_{2}$ und $a_{4}$ liegenden Abschnitte der Geraden $a_{1}$ und $a_{3}$ durch die von ihrem Durchschnittspunkte aus gerechneten Abschnitte der Geraden $a_{2}$ und $a_{4}$ ausdrücken. Man erhält nämlich

$$
\begin{aligned}
& 2 a^{2} b^{\prime} c^{\prime}=\left(b b^{\prime}+c c^{\prime}\right)\left(b c+b^{\prime} c^{\prime}\right)-2 b c\left(b^{\prime 2}+c^{\prime 2}\right), \\
& 2 a^{\prime 2} b c=\left(b b^{\prime}+c c^{\prime}\right)\left(b c+b^{\prime} c^{\prime}\right)-2 b^{\prime} c^{\prime}\left(b^{2}+c^{2}\right) .
\end{aligned}
$$

Jede dieser Gleichungen drückt also die Bedingung der verlangten Proportionalität aus. Wären die Abstände $r$ durch die Seiten und Flächen der beiden in $A^{\prime}$ zusammenstossenden Dreiecke ausgedrückt worden, so hätte man durch völlig analoge, mit den obigen identische Gleichungen die Abschnitte $B B^{\prime}$ und $C C^{\prime}$ der Geraden $a_{2}$ und $a_{4}$ durch die Abschnitte $A^{\prime} B, A^{\prime} B^{\prime}$ $A^{\prime} C, A^{\prime} C^{\prime}$ der Geraden $a_{1}$ und $a_{3}$ ausgedrückt erhalten. Bezeichnet $f$ die Fläche des Vierecks $B C C^{\prime} B^{\prime}$, und wird die Quadratsumme seiner Seiten, $B C^{2}+C C^{\prime 2}+C^{\prime} B^{\prime 2}+B^{\prime} B^{2}=R$ gesetzt, so sind die Abstände $r$ bestimmt durch

$$
R \cdot r_{1}=2 f \cdot B C, \quad R \cdot r_{2}=2 f . C C^{\prime}, \quad R \cdot r_{3}=2 f . C^{\prime} B^{\prime}, \quad R \cdot r_{4}=2 f \cdot B^{\prime} B ;
$$

die Minimalsumme beträgt

$$
r_{1}^{2}+r_{2}^{2}+r_{3}^{2}+r_{4}^{2}=\frac{4 f^{2}}{R} .
$$

2.) Die $r$ sind proportional den Seiten des Vierecks $B A^{\prime} C^{\prime} A$, also

$$
r_{1}: r_{3}: r_{2}: r_{4}=B A^{\prime}: A^{\prime} C^{\prime}: C^{\prime} A: A B \text {. }
$$

Die dazu nöthige Bedingungsgleichung, die wir der Kürze wegen übergehen, drückt auf ganz analoge Weise entweder die Abschnitte $B A^{\prime}$ und $C^{\prime} A$ der Geraden $a_{1}$ und $a_{2}$ durch die Abschnitte $B^{\prime} A, B^{\prime} B, B^{\prime} C^{\prime}, B^{\prime} A^{\prime}$ der Geraden $a_{4}$ und $a_{3}$, oder die Abschnitte $A B$ und $A^{\prime} C^{\prime}$ der Geraden $a_{4}$ und $a_{3}$ durch die Abschnitte $C A^{\prime}, C B, C C^{\prime}, C A$ der Geraden $a_{1}$ und $a_{2}$ aus.

$\mathrm{Zu}$ beiden Aehnlichkeitsfällen wird uns das Sehnenviereck einfache Beispiele liefern.

\$. 9 .

1. Ist $B C C^{\prime} B^{\prime}$ ein Sehnenviereck, dessen Perimeter sich nicht selbst schneidet, so lassen sich, um die Abstände $r$ durch seine Fläche $B C C^{\prime} B^{\prime}=f$ und seine Seiten $B C=a_{1}, C C^{\prime}=a_{2}, C^{\prime} B^{\prime}=a_{3}, B^{\prime} B=a_{t}$ darzustellen, aus 
der Aehnlichkeit der Dreiecke $A C^{\prime} B$ und $A B^{\prime} C$ Werthe von $b, b^{\prime}, c$, $c^{\prime}$, ausgedrückt durch $a_{1}, a_{2}, a_{3}, a_{4}$, herleiten, nämlich

$$
\begin{aligned}
b^{\prime}=a_{3} \frac{a_{1} a_{2}+a_{3} a_{4}}{a_{1}^{2}-a_{3}^{2}}, & c^{\prime}=a_{3} \frac{a_{2} a_{3}+a_{4} a_{1}}{a_{1}^{2}-a_{3}^{2}}, \\
b=a_{1} \frac{a_{2} a_{3}+a_{4} a_{1}}{a_{1}^{2}-a_{3}^{2}}, & c=a_{1} \frac{a_{1} a_{2}+a_{3} a_{4}}{a_{1}^{2}-a_{3}^{2}} .
\end{aligned}
$$

Setzt man diese Werthe in die Gleichungen (2.) und (4.) des $\$$. 8 ein, so erhält man, mit Weglassung des Factors $\frac{a_{1}^{2} a_{3}^{2}}{\left(a_{1}^{2}-a_{3}^{2}\right)^{2}}$,

$$
R=\left(a_{1}^{2}-a_{3}^{2}\right)^{2}+\left(a_{2}^{2}-a_{4}^{2}\right)^{2}+2\left(a_{1} a_{2}+a_{3} a_{4}\right)^{2}+2\left(a_{2} a_{3}+a_{4} a_{1}\right)^{2} .
$$

Berücksichtigt man ferner, dass

$$
2 f=\sin A\left(b c-b^{\prime} c^{\prime}\right)=\frac{\left(a_{1} a_{2}+a_{3} a_{4}\right)\left(a_{2} a_{3}+a_{4} a_{1}\right)}{a_{1}^{2}-a_{3}^{2}} \sin A,
$$

so erhält man $r_{1}, r_{2}, r_{3}, r_{4}$ bestimmt durch die Gleichungen:

$$
\begin{aligned}
& \text { R. } r_{1}=2 f\left[a_{1}\left(a_{1}^{2}+a_{2}^{2}-a_{3}^{2}+a_{4}^{2}\right)+2 a_{2} a_{3} a_{4}\right], \\
& \text { R. } r_{2}=2 f\left[a_{2}\left(a_{2}^{2}+a_{3}^{2}-a_{4}^{2}+a_{1}^{2}\right)+2 a_{3} a_{4} a_{1}\right], \\
& \text { R. } r_{3}=2 f\left[a_{3}\left(a_{3}^{2}+a_{4}^{2}-a_{1}^{2}+a_{2}^{2}\right)+2 a_{4} a_{1} a_{2}\right], \\
& \text { R. } r_{4}=2 f\left[a_{4}\left(a_{4}^{2}+a_{1}^{2}-a_{2}^{2}+a_{3}^{2}\right)+2 a_{1} a_{2} a_{3}\right] .
\end{aligned}
$$

Das Sehnenviereck liefert ein Paar einfache Beispiele zu der im vorigen Paragraphen erwähnten Proportionalität der Abstände $r$ mit den zugehörigen Seiten.

1.) Wenn in einem Sehnenviereck die Producte der gegenüberliegenden Seiten einander gleich sind, so ist der Minimumpunkt der Durchschnitt der Diagonalen, und seine Abstände sind den zugehörigen Seiten proportional. Denn sind $p_{1}, p_{2}, p_{3}, p_{4}$ die Abstände des Diagonalendurchschnittes von den Seiten eines beliebigen Sehnenviereckes, so folgt aus der Aehnlichkeit je zweier gegenüberliegender Dreiecke

und ferner ist

$$
p_{2}: p_{4}=a_{2}: a_{4}, \quad p_{1}: p_{3}=a_{1}: a_{3},
$$

$$
p_{1}: p_{2}=a_{4}: a_{3}, \quad p_{3}: p_{4}=a_{2}: a_{1},
$$

weil diese Verhältnisse die Sinusverhältnisse gleicher Peripheriewinkel sind. Soll aber

folglich

$$
p_{1}: p_{2}=a_{1}: a_{2} \text { und } p_{3}: p_{4}=a_{3}: a_{4}
$$

$$
p_{1}: p_{2}: p_{3}: p_{4}=a_{1}: a_{2}: a_{3}: a_{4}
$$

sein, so wird die Bedingung $a_{1} a_{3}=a_{2} a_{4}$ erfordert. Aus dieser Proportion selbst aber folgt, dass der Diagonalendurchschnitt Minimumpunkt ist (s. \$.2, 3.). 
Um ein solches Sehnenviereck zu construiren, nehme man im Kreise $A B C$ beliebig, verlängere $B C$ rückwärts um $B E=B C$ und mache $\angle C B D=\angle B A E$, so ist $D$ der gesuchte vierte Punkt, wie sogleich aus den ähnlichen Dreiecken $A D C$ und $A B E$ folgt.

Die Werthe der $r$ lassen sich sehr einfach bestimmen; z. B. aus $a_{1} r_{1}+a_{2} r_{2}=a_{1} a_{2} \sin a_{1} a_{2}$ folgt

$$
r_{1}=a_{1} \frac{a_{1}^{2} a_{2} \sin a_{1} a_{2}}{a_{1}^{2}+a_{2}^{2}}, \text { u. s. w. }
$$

2.) In einem Viereck mit zwei gegenüberstehenden rechten Winkeln liegt der Minimumpunkt in der Mitte der Diagonale, welche die rechten Winkel theilt, und seine Entfernungen sind den Seiten des Vierecks mit einspringendem Winkel proportional. Denn sind $B^{\prime}$ und $C$ die rechten Winkel, $O$ die Mitte von $B^{\prime} C$, so ist

$$
\begin{aligned}
& r_{1}=\frac{1}{2} a_{4} \sin B, \quad r_{4}=\frac{1}{2} a_{1} \sin B, \quad r_{2}=\frac{1}{2} a_{3} \sin C^{\prime}, \quad r_{3}=\frac{1}{2} a_{2} \sin C^{\prime}, \\
& a_{1}=B A \cos B, \quad a_{4}=B A^{\prime} \cos B, \quad a_{2}=A^{\prime} C^{\prime} \cos C^{\prime}, a_{3}=A C^{\prime} \cos C^{\prime},
\end{aligned}
$$

wobei unter $C^{\prime}$ der spitze Winkel verstanden wird. Berücksichtigt man, dass $\sin B=\sin C^{\prime}$ und $\cos B=\cos C^{\prime}$ ist, so folgt aus diesen Gleichungen

$$
r_{1}: r_{2}: r_{3}: r_{4}=B A^{\prime}: A^{\prime} C^{\prime}: C^{\prime} A: A B \text {. }
$$

Die $r$ sind also den Seiten des Vierecks $B A^{\prime} C^{\prime} A$ proportional und folglich ist $O$ der Minimumpunkt $(\mathbb{S} .2,3$.). Die Minimalsumme beträgt in diesem Falle

$$
r_{1}^{2}+r_{2}^{2}+r_{3}^{2}+r_{4}^{2}=\frac{1}{4} \sin ^{2} B\left(a_{1}^{2}+a_{2}^{2}+a_{3}^{2}+a_{4}^{2}\right)=\frac{1}{2} \sin ^{2} B . B C^{\prime 2} .
$$

2. Wenn der Perimeter des Sehnenvierecks sich selbst schneidet, so erhält man für die Abstände $r$ ganz analoge, nur durch Vorzeichen verschiedene Formeln. Es mögen also die Punkte $A^{\prime}, C, A, B^{\prime}$ auf einem Kreise liegen, und werde, damit man die Seiten und zugehörigen $r$ der Reihe nach zählt, $A^{\prime} C=a_{1}, C A=a_{2}, A B^{\prime}=a_{3}, B^{\prime} A^{\prime}=a_{4}$ gesetzt. Alsdann ergeben sich aus der Aehnlichkeit der Dreiecke $C C^{\prime} A^{\prime}$ und $B^{\prime} C^{\prime} A, B C A$ und $B B^{\prime} A^{\prime}$ folgende Werthe:

$$
\begin{array}{lll}
b=a_{2}, & c^{\prime}=a_{3}, & a=a_{2} \frac{a_{3} a_{4}-a_{1} a_{2}}{a_{2}^{2}-a_{4}^{2}}, \\
b^{\prime}=a_{3} \frac{a_{2} a_{3}-a_{4} a_{1}}{a_{3}^{2}-a_{1}^{2}}, & c=a_{2} \frac{a_{2} a_{3}-a_{4} a_{1}}{a_{2}^{2}-a_{4}^{2}}, & a^{\prime}=a_{3} \frac{a_{3} a_{4}-a_{1} a_{2}}{a_{3}^{2}-a_{1}^{2}} .
\end{array}
$$

Setzt man diese Werthe in die Gleichungen (4.) des vorigen Paragraphen ein, und berücksichtigt man, dass $2 f=\sin A\left(a_{4} a_{1}-a_{2} a_{3}\right)$, wo $f$ die Fläche $A^{\prime} C A B^{\prime}$ bezeichnet, so erhält man mit Weglassung des Factors $\frac{a_{2}^{2} a_{3}^{2}\left(a_{3} a_{4}-a_{1} a_{2}\right)^{2}}{\left(a_{2}^{2}-a_{4}^{2}\right)^{2}\left(a_{3}^{2}-a_{1}^{2}\right)^{2}}$, 


$$
\begin{aligned}
& \boldsymbol{R}=\left(a_{1}^{2}-a_{3}^{2}\right)^{2}+\left(a_{2}^{2}-a_{4}^{2}\right)^{2}+2\left(a_{1} a_{2}-a_{3} a_{4}\right)^{2}+2\left(a_{2} a_{3}-a_{4} a_{1}\right)^{2}, \\
& R r_{1}=2 f\left[a_{1}\left(a_{1}^{2}+a_{2}^{2}-a_{3}^{2}+a_{4}^{2}\right)-2 a_{2} a_{3} a_{4}\right], \\
& R r_{2}=2 f\left[-a_{2}\left(a_{2}^{2}+a_{3}^{2}-a_{4}^{2}+a_{1}^{2}\right)+2 a_{3} a_{4} a_{1}\right], \\
& R r_{3}=2 f\left[-a_{3}\left(a_{3}^{2}+a_{4}^{2}-a_{1}^{2}+a_{2}^{2}\right)+2 a_{4} a_{1} a_{2}\right], \\
& R r_{4}=2 f\left[a_{4}\left(a_{4}^{2}+a_{1}^{2}-a_{2}^{2}+a_{3}^{2}\right)-2 a_{1} a_{2} a_{3}\right] .
\end{aligned}
$$

Der besondere Fall, dass die Winkel bei $B^{\prime}$ und bei $C$ rechte sind, also $\boldsymbol{A} \boldsymbol{A}^{\prime}$ ein Kreisdurchmesser, fällt mit dem vorhin erwähnten eines Vierecks mit zwei gegenüberstehenden rechten Winkeln zusammen; $O$ ist die Mitte von $B^{\prime} C$. Die Ausdrücke durch die Seiten des Vierecks $A^{\prime} C A B^{\prime}$ sind:

$$
\begin{gathered}
r_{1}=\frac{1}{2} a_{4} \sin A, \quad r_{2}=\frac{1}{2} a_{3} \sin A, \quad r_{3}=\frac{1}{2} a_{2} \sin A, \quad r_{4}=\frac{1}{2} a_{1} \sin A, \\
r_{1}: r_{2}: r_{3}: r_{4}=a_{4}: a_{3}: a_{2}: a_{1}, \\
r_{1}^{2}+r_{2}^{2}+r_{3}^{2}+r_{4}^{2}=\frac{1}{4} \sin ^{2} A\left(a_{1}^{2}+a_{2}^{2}+a_{3}^{2}+a_{4}^{2}\right)=\frac{1}{2} A^{\prime} A^{2} \sin ^{2} A .
\end{gathered}
$$

3. Wenn $B C C^{\prime} B^{\prime}$ ein Paralleltrapez mit den parallelen Seiten $B C$ und $B^{\prime} C^{\prime}$ ist, und die Abstände $r$ durch $a, b, c$ und die Höhen $A H=h$ und $A H^{\prime}=h^{\prime}$ ausgedrückt werden sollen, so hat man in die Gleichungen (2.) und (4.) des vorigen Paragraphen $\frac{b}{b^{\prime}}=\frac{c}{c^{\prime}}=\frac{a}{a^{\prime}}=\frac{h}{h^{\prime}}$ einzusetzen und erhält dadurch, mit Weglassung des Factors $a^{\prime 2}$,

$$
\begin{aligned}
& \boldsymbol{R}=\boldsymbol{a}^{2}+2\left(b^{2}+c^{2}\right), \\
& \boldsymbol{R} r_{1}=h^{\prime}\left(a^{2}+b^{2}+c^{2}\right)-h^{\prime}\left(b^{2}+c^{2}\right), \\
& \boldsymbol{R} r_{3}=h\left(b^{2}+c^{2}\right)-h^{\prime}\left(a^{2}+b^{2}+c^{2}\right), \\
& \boldsymbol{R} r_{2}=a b\left(h+h^{\prime}\right), \\
& \boldsymbol{R} r_{4}=a c\left(h+h^{\prime}\right) .
\end{aligned}
$$

Hieraus folgt $r_{2}: r_{4}=b: c ; O$ liegt also auf der durch $A$ gehenden Axe des

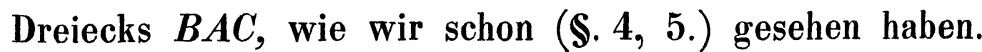

Führt man dagegen die Seiten des Paralleltrapezes, $B C=a_{1}, C C^{\prime}=a_{2}$, $C^{\prime} B^{\prime}=a_{3}, B^{\prime} B=a_{4}$ und seine Höhe $h-h^{\prime}=k=h \frac{a_{1}-a_{3}}{a_{1}}$ ein, so erhält man

$$
\begin{aligned}
& \boldsymbol{R}=2\left(a_{2}^{2}+a_{4}^{2}\right)+\left(a_{1}-a_{3}\right)^{2}, \\
& \text { R. } r_{1}=k\left[a_{2}^{2}+a_{4}^{2}+a_{1}^{2}-a_{1} a_{3}\right], \\
& \text { R. } r_{3}=k\left[a_{2}^{2}+a_{4}^{2}+a_{3}^{2}-a_{1} a_{3}\right], \\
& \text { R. } r_{2}=k a_{2}\left(a_{1}+a_{3}\right), \\
& \text { R. } r_{4}=k a_{4}\left(a_{1}+a_{3}\right)
\end{aligned}
$$

Beim Paralleltrapez kann der erste der im vorigen Paragraphen erwähnten 
Aehnlichkeitsfälle eintreten. Wenn nämlich das doppelte Rechteck der parallelen Seiten gleich der Quadratsumme der nicht parallelen ist, so sind die Abstände $r$ den zugehörigen Seiten proportional, und ihre Quadratsumme ist gleich dem Quadrate der Trapezhöhe. Denn die Bedingung

ergiebt die Werthe

$$
a_{2}^{2}+a_{4}^{2}=2 a_{1} a_{3}
$$

$$
r_{1}=k \frac{a_{1}}{a_{1}+a_{3}}, \quad r_{2}=k \frac{a_{2}}{a_{1}+a_{3}}, \quad r_{3}=k \frac{a_{3}}{a_{1}+a_{3}}, \quad r_{4}=k \frac{a_{4}}{a_{1}+a_{3}},
$$

folglich

$$
\begin{gathered}
r_{1}: r_{2}: r_{3}: r_{4}=a_{1}: a_{2}: a_{3}: a_{4}, \\
r_{1}^{2}+r_{2}^{2}+r_{3}^{2}+r_{4}^{2}=k^{2} .
\end{gathered}
$$

§. 10.

Auf dieselbe Weise, wie bei vier Geraden, gelangt man bei beliebig vielen Geraden in der Ebene dazu, die Abstände $r$ des Minimumpunktes durch die Seiten und Flächen der in einem der Durchschnittspunkte der Geraden zusammenstossenden Dreiecke auszudrücken; der gemeinschaftliche Nenner der $r$ ist eine symmetrische Determinante, deren Grad gleich der Anzahl der Geraden ist. Es werde dies noch an fünf Geraden gezeigt.

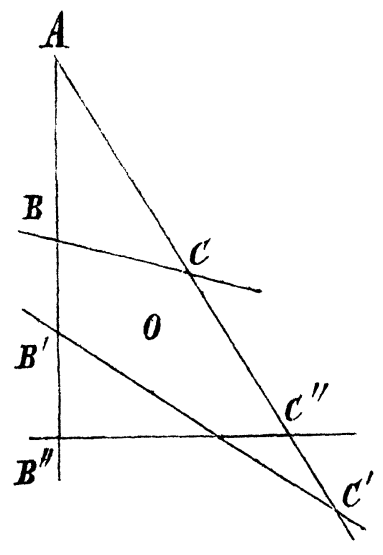

Auf zwei der gegebenen Geraden, die sich im Punkte $A$ schneiden, werden von den drei übrigen die Abschnitte gebildet: $A B=c, A C=b, A B^{\prime}=c^{\prime}, A C^{\prime}=b^{\prime}$, $A B^{\prime \prime}=c^{\prime \prime}, A C^{\prime \prime}=b^{\prime \prime}$, während jene auf diesen dreien die Abschnitte $B C=a, B^{\prime} C^{\prime}=a^{\prime}, B^{\prime \prime} C^{\prime \prime}=a^{\prime \prime}$ bilden. Ferner seien $r_{1}, r_{2}, r_{3}, r_{4}, r_{5}$ der Reihe nach die Abstände von $a, a^{\prime}, a^{\prime \prime}, b, c$.

Indem man in der Gleichung $\Sigma r \sin \alpha=0$ einmal $r_{4}$, dann $r_{5}$ als Nullinie nimmt, erhält man $r_{1} \sin C+r_{5} \sin A-r_{2} \sin C^{\prime}-r_{3} \sin C^{\prime \prime}=0$, $r_{1} \sin B+r_{4} \sin A-r_{3} \sin B^{\prime \prime}-r_{2} \sin B^{\prime}=0$.

Setzt man nach Division mit $\sin A$ statt $\operatorname{der}$ Sinus die ihnen proportionalen Seiten, so folgt

$$
\begin{aligned}
& r_{1} a^{\prime} a^{\prime \prime} c-r_{2} a a^{\prime \prime} c^{\prime}-r_{3} a a^{\prime} c^{\prime \prime}+r_{5} a a^{\prime} a^{\prime \prime}=0, \\
& r_{1} a^{\prime} a^{\prime \prime} b-r_{2} a a^{\prime \prime} b^{\prime}-r_{3} a a^{\prime} b^{\prime \prime}+r_{4} a a^{\prime} a^{\prime \prime}=0 .
\end{aligned}
$$

Dazu kommen noch drei Gleichungen, welche die Flächen $A B C=f, A B^{\prime} C^{\prime}=f^{\prime}$, 
$A B^{\prime \prime} C^{\prime \prime}=f^{\prime \prime}$ ausdrücken, so dass man zur Bestimmung der $r$ folgende Gleichungen hat:

$$
\begin{array}{rrrrrr}
a r_{1} & & -b r_{4} & -c r_{5} & = & -2 f, \\
a^{\prime} r_{2} & & +b^{\prime} r_{4} & +c^{\prime} r_{5} & = & 2 f^{\prime}, \\
& a^{\prime \prime} r_{3} & +b^{\prime \prime} r_{4} & +c^{\prime \prime} r_{5} & = & 2 f^{\prime \prime}, \\
-a^{\prime} a^{\prime \prime} b r_{1} & +a a^{\prime \prime} b^{\prime} r_{2} & +a a^{\prime} b^{\prime \prime} r_{3} & -a a^{\prime} a^{\prime \prime} r_{4} & & =0, \\
-a^{\prime} a^{\prime \prime} c r_{1}+a a^{\prime \prime} c^{\prime} r_{2} & +a a^{\prime} c^{\prime \prime} r_{3} & & -a a^{\prime} a^{\prime \prime} r_{5} & = & 0 .
\end{array}
$$

Der gemeinschaftliche Nenner der $r$ wird daher durch folgende symmetrische Determinante dargestellt:

$$
a^{2} a^{\prime 2} a^{\prime \prime 2}\left|\begin{array}{ccccc}
\frac{a}{a^{\prime} a^{\prime \prime}} & 0 & 0 & -b & -c \\
0 & \frac{a^{\prime}}{a a^{\prime \prime}} & 0 & b^{\prime} & c^{\prime} \\
0 & 0 & \frac{a^{\prime \prime}}{a a^{\prime}} & b^{\prime \prime} & c^{\prime \prime} \\
-b & b^{\prime} & b^{\prime \prime} & -a a^{\prime} a^{\prime \prime} & 0 \\
-c & c^{\prime} & c^{\prime \prime} & 0 & -a a^{\prime} a^{\prime \prime}
\end{array}\right|=a a^{\prime} a^{\prime \prime} R,
$$

wo $\boldsymbol{R}$ folgenden Werth hat:

$$
\begin{aligned}
R= & a^{2} a^{\prime 2} a^{\prime 2} \\
& +a^{2} a^{\prime 2}\left(b^{\prime 2}+c^{\prime 2}\right)+a^{\prime 2} a^{\prime 2}\left(b^{2}+c^{2}\right)+a^{\prime \prime 2} a^{2}\left(b^{\prime 2}+c^{\prime 2}\right) \\
& +a^{2}\left(b^{\prime} c^{\prime \prime}-b^{\prime \prime} c^{\prime}\right)^{2}+a^{\prime 2}\left(b c^{\prime \prime}-b^{\prime \prime} c\right)^{2}+a^{\prime 2}\left(b c^{\prime}-b^{\prime} c\right)^{2} .
\end{aligned}
$$

\$. 11.

In Bezug auf vier Ebenen, welche eine dreiseitige Pyramide $A_{1} A_{2} A_{3} A_{4}$ mit den den gleichbezeichneten Eckpunkten gegenüberliegenden Seitenflächen $f_{1}, f_{2}, f_{3}, f_{4}$ begrenzen, haben wir in $\$ .5,1$. gesehen, dass ihr Minimumpunkt sich als der gemeinschaftliche Durchschnittspunkt von vier Geraden $A_{1} O_{1}$, $A_{2} O_{2}, \ldots$ bestimmt, deren jede eine Ecke mit dem Minimumpunkte der gegenüberliegenden Fläche verbindet; dass ferner $O_{1}, O_{2}, \ldots$ Scheitel von je drei auf den Pyramidenkanten stehenden Dreiecken sind, die sich wie die Quadrate der anliegenden Pyramidenflächen verhalten; endlich, dass die Abstände $r$ den zugehörigen Flächen proportional sind, $r_{1}: r_{2}: r_{3}: r_{4}=f_{1}: f_{2}: f_{3}: f_{4}$. Es ist noch hinzuzufügen, dass $O$ die gemeinschaftliche Spitze von vier Pyramiden ist, deren Volumina sich wie die Quadrate der gegebenen Pyramidenflächen verhalten, auf denen sie stehen, z. B. $O A_{1} A_{2} A_{3}: O A_{2} A_{3} A_{4}=f_{4} r_{4}: f_{1} r_{1}=f_{4}^{2}: f_{1}^{2}$. 
Bezeichnet man mit $h_{1}, h_{2}, h_{3}, h_{4}$ die Höhen der Pyramide, mit $V$ ihr Volumen, mit $R$ die Quadratsumme ihrer Seitenflächen, $f_{1}^{2}+f_{2}^{2}+f_{3}^{2}+f_{4}^{2}$, so ist

$$
3 V=f_{1} r_{1}+f_{2} r_{2}+f_{3} r_{3}+f_{4} r_{4}=f_{1} h_{1}=f_{2} h_{2}=f_{3} h_{3}=f_{4} h_{4},
$$

folglich, da die $r$ den zugehörigen Seitenflächen proportional sind,

$$
\boldsymbol{R} . r_{1}=h_{1} f_{1}^{2}, \quad \boldsymbol{R} . r_{2}=h_{2} f_{2}^{2}, \quad R \cdot r_{3}=h_{3} f_{3}^{2}, \quad R . r_{4}=h_{4} f_{4}^{2},
$$

woraus sich als Minimalsumme der Quadrate der Abstände ergiebt:

$$
r_{1}^{2}+r_{2}^{2}+r_{3}^{2}+r_{4}^{2}=\frac{9 V^{2}}{R} \text {. }
$$

Wenn drei Ebenen der Pyramide, $f_{1}, f_{2}, f_{3}$, sich rechtwinklig schneiden, in $A_{4}$ also drei rechte Kantenwinkel zusammenstossen, so ist $O_{4}$ der gemeinschaftliche Durchschnittspunkt der drei Höhen des Dreiecks $A_{1} A_{2} A_{3}$, die Axe $A_{4} O_{4}$ ist die zur Ebene $f_{4}$ gehörige Pyramidenhöhe $h_{4}$, der Minimumpunkt $O$ ist deren Mitte, und die Minimalsumme der Quadratabstände ist dem halben Quadrate dieser Höhe gleich. Denn nach $\$ .5$ ist

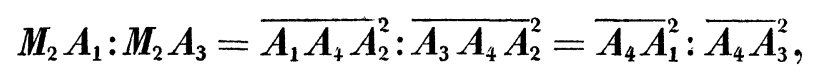

folglich steht $A_{4} M_{2}$ in dem rechtwinkligen Dreieck $A_{1} A_{4} A_{3}$ auf der Hypotenuse $A_{1} A_{3}$ serikrecht. Da ferner $A_{4} A_{2}$ auf der Ebene $A_{1} A_{4} A_{3}$ senkrecht steht, so ist die Ebene $A_{4} M_{2} A_{2}$ zu $A_{1} A_{3}$, also auch $A_{2} M_{2}$ zu $A_{1} A_{3}$ senkrecht. Gleiches gilt von $A_{3} M_{3}$ und $A_{1} M_{1}$ in Bezug auf $A_{1} A_{2}$ und $A_{2} A_{3}$. Wegen $f_{4}^{2}=f_{1}^{2}+f_{2}^{2}+f_{3}^{2}$ folgt $r_{4}=\frac{1}{2} h_{4}^{2}$, und wegen $r_{1}^{2}+r_{2}^{2}+r_{3}^{2}=\overline{O A}_{4}^{2}=\frac{1}{4} h_{4}^{2}$ ergiebt sich $r_{1}^{2}+r_{2}^{2}+r_{3}^{2}+r_{4}^{2}=\frac{1}{2} h_{4}^{2}$. Noch ist hinzuzufügen, dass von den vier Pyramiden, deren Spitze $O$ ist, die über $A_{1} A_{2} A_{3}$ gleich der Summe der übrigen drei ist, weil sie den Quadraten der Flächen, auf denen sie stehen, proportional sind. Alle diese Beziehungen sind denen beim rechtwinkligen Dreieck (s. \$. 7) vollständig analog.

Um die Analogie des Falles von vier Ebenen mit dem einer grösseren Anzahl zu zeigen, werde noch bemerkt, dass man zur Bestimmung der $r$ folgendes Gleichungssystem aufstellen kann:

$$
\begin{aligned}
f_{4} \cdot r_{1} & -f_{1} \cdot r_{4}=0, \\
f_{4} \cdot r_{2} & -f_{2} \cdot r_{4}=0, \\
f_{4} \cdot r_{3}-f_{3} \cdot r_{4} & =0, \\
f_{1} \cdot r_{1}+f_{2} \cdot r_{2}+f_{3} \cdot r_{3}+f_{4} \cdot r_{4} & =3 V,
\end{aligned}
$$

so dass der gemeinschaftliche Nenner der $r$ durch folgende symmetrische 
Determinante dargestellt wird:

$$
-\left|\begin{array}{rrrr}
f_{4} & 0 & 0 & f_{1} \\
0 & f_{4} & 0 & f_{2} \\
0 & 0 & f_{4} & f_{3} \\
f_{1} & f_{2} & f_{3} & -f_{4}
\end{array}\right|=f_{4}\left(f_{1}^{2}+f_{2}^{2}+f_{3}^{2}+f_{4}^{2}\right)
$$

S. 12.

Sind beliebige Ebenen im Raume gegeben, so kann man die Abstände $r$ ihres Minimumpunktes durch die Seitenflächen und Volumina der in einem der Durchschnittspunkte zusammenstossenden dreiseitigen Pyramiden ausdrücken. Wir wollen dies an fünf Ebenen zeigen; dieselben bilden das Analogon zu vier Geraden in der Ebene.

Es mögen in beistehender Figur die Seitenflächen wie folgt bezeichnet werden: $B C D=a$, $B^{\prime} C^{\prime} D^{\prime}=a^{\prime}, A C D=b, A C^{\prime} D^{\prime}=b^{\prime}, A D B=c$, $A D^{\prime} B^{\prime}=c^{\prime}, A B C=d, A B^{\prime} C^{\prime}=d^{\prime} ;$ und zu den Ebenen $b, c, d, a, a^{\prime}$ mögen der Reihe nach die Abstände $r_{1}, r_{2}, r_{3}, r_{4}, r_{5}$ gehören. Die Bedingung $\Sigma r \cos \alpha=0$ muss in Bezug auf wenigstens drei durch $O$ gelegte Gerade erfüllt sein, als solche nehmen wir Parallelen zu $A B, A C, A D$. In Bezug auf eine Parallele zu $A B$ geht, weil $r_{2}$ und $r_{3}$ auf $A B$ senkrecht stehen, die Bedingung

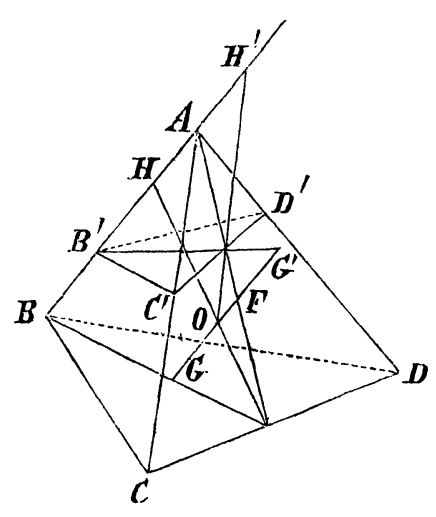
$\Sigma r \cos \alpha=0$ über in

$$
r_{1} \cos \alpha_{1}+r_{4} \cos \alpha_{4}+r_{5} \cos \alpha_{5}=0 \text {. }
$$

Man kann nun aber die Verhältnisse $\cos \alpha_{4}: \cos \alpha_{1}$ und $\cos \alpha_{5}: \cos \alpha_{1}$ durch die gegebenen Seitenflächen ausdrücken; denn man hat einerseits

$$
O C D A: O C D B=b r_{1}: a r_{4} \text {. }
$$

Schneidet die durch $O$ gelegte Parallele zu $A B$ die Fläche $C D B$ in $G, C D A$ in $F$, schneidet die Ebene $O C D$ die Linie $A B$ in $H$, so ist andererseits, weil jene Pyramiden die gemeinschaftliche Grundfläche $O C D$ haben, ihr Verhältniss gleich dem der Höhen von $A$ und $B$ auf $O C D$, folglich gleich dem der $A b-$ schnitte $H A$ und $H B$, also

$$
O C D A: O C D B=H A: H B=O F: O G=-\frac{r_{1}}{\cos \alpha_{1}}: \frac{r_{4}}{\cos \alpha_{4}} .
$$


Aus dieser Gleichung folgt in Verbindung mit der vorhergehenden

$$
\frac{\cos \alpha_{4}}{\cos \alpha_{1}}=-\frac{b}{a} \text {. }
$$

Wird ferner $B^{\prime} C^{\prime} D^{\prime}$ von $F G$ in $G^{\prime}$ und $O C^{\prime} D^{\prime}$ von $A B$ in $H^{\prime}$ geschnitten, so ist folglich

$$
O C^{\prime} D^{\prime} A: O C^{\prime} D^{\prime} B^{\prime}=b^{\prime} r_{1}: a^{\prime} r_{5}=H^{\prime} A: H^{\prime} B^{\prime}=O F: O G^{\prime}=\frac{r_{1}}{\cos \alpha_{1}}: \frac{r_{5}}{\cos \alpha_{5}},
$$

$$
\frac{\cos \alpha_{5}}{\cos \alpha_{1}}=\frac{b^{\prime}}{a^{\prime}}
$$

Setzt man diese Werthe in die Bedingungsgleichung ein, so erhält man

$$
r_{1} a a^{\prime}-r_{4} a^{\prime} b+r_{5} a b^{\prime}=0 \text {. }
$$

Nimmt man ein zweites Mal als Nulllinie eine Parallele zu $A C$ und ein drittes Mal eine solche zu $A D$, so folgt in gleicher Weise

$$
\begin{aligned}
& r_{2} a a^{\prime}-r_{4} a^{\prime} c+r_{5} a c^{\prime}=0, \\
& r_{3} a a^{\prime}-r_{4} a^{\prime} d+r_{5} a d^{\prime}=0 .
\end{aligned}
$$

Dazu kommen noch, wenn man $A B C D=v$ und $A B^{\prime} C^{\prime} D^{\prime}=v^{\prime}$ setzt,

$$
\begin{aligned}
b r_{1}+c r_{2}+d r_{3}+a r_{4} & =3 v, \\
b^{\prime} r_{1}+c^{\prime} r_{2}+d^{\prime} r_{3}-a^{\prime} r_{5} & =3 v^{\prime} .
\end{aligned}
$$

Daher sind die Abstände $r$ durch folgendes Gleichungssystem bestimmt:

$$
\begin{array}{rlrl}
a a^{\prime} r_{1} & -a^{\prime} b r_{4}+a b^{\prime} r_{5} & =0, \\
& a a^{\prime} r_{2} & -a^{\prime} c r_{4}+a c^{\prime} r_{5} & =0, \\
& a a^{\prime} r_{3} & -a^{\prime} d r_{4}+a d^{\prime} r_{5} & =0, \\
-b r_{1}-c r_{2}-d r_{3}-a r_{4} & & =-3 v, \\
b^{\prime} r_{1}+c^{\prime} r_{2}+d^{\prime} r_{3} & -a^{\prime} r_{5} & =3 v^{\prime} .
\end{array}
$$

Der gemeinschaftliche Nenner der $r$ wird daher durch die symmetrische Determinante dargestellt:

$$
a a^{\prime}\left|\begin{array}{ccccc}
a a^{\prime} & 0 & 0 & -b & b^{\prime} \\
0 & a a^{\prime} & 0 & -c & c^{\prime} \\
0 & 0 & a a^{\prime} & -d & d^{\prime} \\
-b & -c & -d & -\frac{a}{a^{\prime}} & 0 \\
b^{\prime} & c^{\prime} & d^{\prime} & 0 & -\frac{a^{\prime}}{a}
\end{array}\right|=a^{2} a^{\prime 2} R
$$


wo $R$ den Werth hat:

$R=a^{2} a^{\prime 2}+a^{2}\left(b^{\prime 2}+c^{\prime 2}+d^{\prime 2}\right)+a^{\prime 2}\left(b^{2}+c^{2}+d^{2}\right)+\left(b c^{\prime}-b^{\prime} c\right)^{2}+\left(c d^{\prime}-c^{\prime} d\right)^{2}+\left(d b^{\prime}-d^{\prime} b\right)^{2}$.

Die Abstände $r$ sind durch folgende Gleichungen bestimmt:

$$
\begin{aligned}
& R . r_{1}=3 v\left[b\left(a^{\prime 2}+c^{\prime 2}+d^{\prime 2}\right)-b^{\prime}\left(c c^{\prime}+d d^{\prime}\right)\right]+3 v^{\prime}\left[b^{\prime}\left(a^{2}+c^{2}+d^{2}\right)-b\left(c c^{\prime}+d d^{\prime}\right)\right], \\
& R . r_{2}=3 v\left[c\left(a^{\prime 2}+b^{\prime 2}+d^{\prime 2}\right)-c^{\prime}\left(d d^{\prime}+b b^{\prime}\right)\right]+3 v^{\prime}\left[c^{\prime}\left(a^{2}+b^{2}+d^{2}\right)-c\left(d d^{\prime}+b b^{\prime}\right)\right], \\
& R . r_{3}=3 v\left[d\left(a^{\prime 2}+b^{\prime 2}+c^{\prime 2}\right)-d^{\prime}\left(b b^{\prime}+c c^{\prime}\right)\right]+3 v^{\prime}\left[d^{\prime}\left(a^{2}+b^{2}+c^{2}\right)-d\left(b b^{\prime}+c c^{\prime}\right)\right], \\
& R . r_{4}=a\left[3 v\left(a^{\prime 2}+b^{\prime 2}+c^{\prime 2}+d^{\prime 2}\right)-3 v^{\prime}\left(b b^{\prime}+c c^{\prime}+d d^{\prime}\right)\right], \\
& R . r_{5}=a^{\prime}\left[3 v\left(b b^{\prime}+c c^{\prime}+d d^{\prime}\right)-3 v^{\prime}\left(a^{2}+b^{2}+c^{2}+d^{2}\right)\right] .
\end{aligned}
$$

Der Vergleich zeigt die Analogie dieser Formeln mit denen für vier Gerade (\$.8).

Sind zwei Ebenen, $a$ und $a^{\prime}$, parallel, so dass die gegebenen fünf Ebenen eine parallel abgestumpfte dreiseitige Pyramide begrenzen, so hat man $\frac{a^{\prime}}{a}=\frac{b^{\prime}}{b}=\frac{c^{\prime}}{c}=\frac{d^{\prime}}{d}$ zu setzen, wodurch man für $R$, mit Weglassung des Factors $a^{\prime 2}$, den Werth

$$
R=a^{2}+2\left(b^{2}+c^{2}+d^{2}\right)
$$

erhält. Bezeichnen ferner $h$ und $h^{\prime}$ die zu $a$ und $a^{\prime}$ gehörigen Höhen, so ist $3 v=a h$ und $3 v^{\prime}=a^{\prime} h^{\prime}$, und man erhält

$$
\begin{aligned}
& \boldsymbol{R} . r_{1}=a b\left(h+h^{\prime}\right), \\
& \boldsymbol{R} \cdot r_{2}=a c\left(h+h^{\prime}\right), \\
& \boldsymbol{R} . r_{3}=a d\left(h+h^{\prime}\right), \\
& \boldsymbol{R} \cdot r_{4}=h\left(a^{2}+b^{2}+c^{2}+d^{2}\right)-h^{\prime}\left(b^{2}+c^{2}+d^{2}\right), \\
& \boldsymbol{R} \cdot r_{5}=h\left(b^{2}+c^{2}+d^{2}\right)-h^{\prime}\left(a^{2}+b^{2}+c^{2}+d^{2}\right) .
\end{aligned}
$$

Diese Formeln sind denen für das Paralleltrapez ganz analog. Da $r_{1}: r_{2}: r_{3}=b: c: d$, so liegt $O$ auf $\operatorname{der} \operatorname{durch} A$ gehenden Axe von $a, b, c$, $d$, wie wir schon (\$. 5) gesehen haben.

\section{Ueber das Minimum oder Maximum der Potenzsumme der Abstände eines Punktes von gegebenen Punkten.}

\$. 13.

1. Wird mit $r$ der Abstand eines der gegebenen Punkte $A$ von einem beliebigen Punkte $O$, mit $r^{\prime}$ sein Abstand von einem anderen beliebigen 
Punkte $O^{\prime}$, ferner $O O^{\prime}$ mit $t$ bezeichnet, so ist

$$
r^{\prime 2}=r^{2}-2 r t \cos r t+t^{2}=r^{2}\left(1-2 t \frac{\cos r t}{r}+\frac{1}{r^{2}} t^{2}\right),
$$

woraus durch Potenzirung mit einer beliebigen Zahl $\frac{n}{2}$ und Entwickelung nach dem binomischen Satze folgt:

$$
\begin{aligned}
r^{\prime n} & =r^{n}\left[1-\left(2 t \frac{\cos r t}{r}-\frac{1}{r^{2}} t^{2}\right)\right]^{\frac{n}{2}} \\
& =r^{n}\left[1-n t \frac{\cos r t}{r}+\frac{n}{2} t^{2} \frac{1+(n-2) \cos ^{2} r t}{r^{2}}-\cdots\right] .
\end{aligned}
$$

Bildet man diese Gleichung für jeden der Punkte $A$, so erhält man durch Summation

$$
\Sigma r^{\prime n}=\Sigma r^{n}-n t \Sigma r^{n-1} \cos r t+\frac{n}{2} t^{2} \Sigma r^{n-2}\left(1+(n-2) \cos ^{2} r t\right)-\cdots
$$

Wählt man daher den Punkt $O$ so, dass die Gleichung

$$
\text { (1.) } \Sigma r^{n-1} \cos r t=0
$$

erfüllt ist, so hängt das Wachsthum von $\Sigma r^{n}$ nur von dem Vorzeichen des Ausdruckes

$$
\frac{n}{2} \sum r^{n-2}\left(1+(n-2) \cos ^{2} r t\right)
$$

ab, da $t$ stets so klein genommen werden kann, dass das Glied der Entwickelung, welches den Factor $t^{2}$ enthält, alle folgenden Glieder überwiegt. Ist also die Summe der Projectionen der $n-1^{\text {ten }}$ Potenzen der Abstände auf jede Gerade durch $O$ gleich Null, oder, was dasselbe, geben diese $n-1^{\text {ten }}$ Potenzen, der Grösse und Richtung nach an einander gesetzt, ein geschlossenes Vieleck, so ist, wenn man sich $t$ als eine unendlich kleine Grösse erster Ordnung denkt, das Increment $\varepsilon$ von $\Sigma r^{n}$ eine solche zweiter Ordnung.

Diese Bedingung $\Sigma r^{n-1} \cos r t=0$, muss in Bezug auf zwei Gerade einer Ebene erfüllt sein, um für jede Gerade der Ebene, und in Bezug auf drei nicht in einer Ebene liegende Gerade, um für jede Gerade in Raume zu gelten. Soll $O$ auf einer vorgeschriebenen Geraden oder Ebene liegen, so muss jene Bedingung in Bezug auf diese Gerade oder in Bezug auf jede Gerade dieser Ebene erfüllt sein; setzt man die Grössen $r^{n-1}$ der Grösse und Richtung nach an einander, so müssen Anfangspunkt und Endpunkt in einer Normalen zur gegebenen Geraden oder Ebene liegen. 
Wetzig, Potenssumme der Abstände von Punkten, Geraden, Ebenen.

Aus Gleichung (1.) lässt sich eine bemerkenswerthe Relation ableiten. Es ist

$$
\begin{aligned}
r^{\prime 2} & =r^{2}-2 r t \cos r t+t^{2}, \\
r^{\prime 2} \cdot r^{n-2} & =r^{n}+t^{2} \cdot r^{n-2}-2 r^{n-1} t \cos r t, \\
\Sigma r^{\prime 2} \cdot r^{n-2} & =\Sigma r^{n}+t^{2} \Sigma r^{n-2} .
\end{aligned}
$$

Es ist mithin für alle Punkte einer Kugelfläche mit dem Mittelpunkte $O$ die Productensumme $\Sigma r^{\prime 2} \cdot r^{n-2}$ constant und ihre Zunahme dem Quadrate des Kugelradius proportional. Für $n=\mathbf{2}$ folgt, wenn $k$ die Anzahl der Punkte bedeutet, die bekannte Gleichung

$$
\Sigma r^{\prime 2}=\Sigma r^{2}+k t^{2}
$$

2. Das Increment

$$
\text { (2.) } \varepsilon=\frac{n}{2} t^{2} \Sigma r^{n-2}\left(1+(n-2) \cos ^{2} r t\right)
$$

ist offenbar für jedes $n \geqq 1$ positiv; für den Punkt $O$ ist also die Potenzsumme der Abstände, $\Sigma r^{n}$, nach jeder Richtung Minimum, wenn $n$ grösser als die positive Einheit oder ihr gleich ist ${ }^{*}$ ).

Dagegen kann für $n<1 \varepsilon$ negativ werden, also auch entweder ein relatives Maximum und Minimum oder ein absolutes Maximum eintreten.

Zunächst ist nun zu bemerken, dass ein absolutes Maximum nur für $-1<n<0$ möglich ist. Denn bildet man nach Gleichung (2.) die Summe der Incremente $\varepsilon^{\prime}, \varepsilon^{\prime \prime}$, $\varepsilon^{\prime \prime \prime}$ nach beliebigen drei auf einander senkrechten Richtungen $t^{\prime}, t^{\prime \prime}, t^{\prime \prime \prime}$, so erhält man, weil

$$
\begin{aligned}
& \cos ^{2} r t^{\prime}+\cos ^{2} r t^{\prime \prime}+\cos ^{2} r t^{\prime \prime \prime}=1, \\
& \varepsilon^{\prime}+\varepsilon^{\prime \prime}+\varepsilon^{\prime \prime \prime}=\frac{n(n+1)}{2} t^{2} \Sigma r^{n-2},
\end{aligned}
$$

also eine von der Richtung unabhängige und mit Ausnahme des Falles $-1<n<0$ stets positive Grösse. Dagegen muss für $n=-1$, wo diese Summe gleich Null ist, stets relatives Maximum und Minimum stattinden, wenn nicht $\varepsilon$ selbst identisch verschwindet.

Sind $x, y, z$ drei durch $O$ gelegte rechtwinklige Axen, und bezeichnet man die Winkel, die $t$ und $r$ mit ihnen bilden, durch

so ist

$$
\begin{aligned}
& t x=\xi, \quad t y=\eta, \quad t z=\zeta, \\
& r x=\alpha, \quad r y=\beta, \quad r z=\gamma,
\end{aligned}
$$

$$
\cos r t=\cos \xi \cos \alpha+\cos \eta \cos \beta+\cos \zeta \cos \gamma \text {. }
$$

*) Die Bedingung $\Sigma \cos r t=0$ im Falle $n=1$ haben schon Tédénat in Gergonnes Annalen Band 1, p. 285 und L'huilier ebendaselbst p. 297 auf anderem Wege gefunden, wobei jedoch unentschieden bleibt, ob Minimum oder Maximum stattfindet. 
Durch Einsetzung dieses Werthes in Gleichung (2.) ergiebt sich

$$
\left\{\begin{array}{c}
\varepsilon=\frac{n}{2} t^{2}\left\{\Sigma r^{n-2}+(n-2)\left[\cos ^{2} \xi \Sigma r^{n-2} \cos ^{2} \alpha+\cos ^{2} \eta \Sigma r^{n-2} \cos ^{2} \beta+\cos ^{2} \zeta \Sigma r^{n-2} \cos ^{2} \gamma\right.\right. \\
+2 \cos \xi \cos \eta \Sigma r^{n-2} \cos \alpha \cos \beta+2 \cos \eta \cos \zeta \Sigma r^{n-2} \cos \beta \cos \gamma \\
\left.\left.+2 \cos \zeta \cos \xi \Sigma r^{n-2} \cos \gamma \cos \alpha\right]\right\} .
\end{array}\right.
$$

Wählt man die Coordinatenaxen so, dass die drei letzten Glieder der eckigen Klammer verschwinden - was stets möglich ist, wie man aus der Discussion der allgemeinen Gleichung der Flächen zweiten Grades weiss - , so erhält $\varepsilon$ die Form

$$
\text { (4.) } \varepsilon=t^{2}\left(A \cos ^{2} \xi+B \cos ^{2} \eta+C \cos ^{2} \zeta\right) \text {, }
$$

wo

$$
\begin{aligned}
A & =\frac{n}{2} \Sigma r^{n-2}\left(1+(n-2) \cos ^{2} \alpha\right), \\
B & =\frac{n}{2} \Sigma r^{n-2}\left(1+(n-2) \cos ^{2} \beta\right), \\
C & =\frac{n}{2} \Sigma r^{n-2}\left(1+(n-2) \cos ^{2} \gamma\right) .
\end{aligned}
$$

Es giebt daher in dem Systeme der Punkte drei auf einander senkrechte Axen und nach den Richtungen derselben drei Hauptincremente $A t^{2}, B t^{2}, C t^{2}$, aus denen man das Increment nach einer beliebigen Richtung durch Gleichung (4.), also als reciproken Quadratvector einer centralen Fläche zweiten Grades, d.i. als Quadratvector ihrer Fusspunktenfläche aus dem Mitttelpunkte findet; oder, was dasselbe, die Punkte constanten Incrementes liegen auf einer centralen Fläche zweiten Grades, deren Mittelpunkt $O$ ist.

Ist jeder der Coefficienten $A, B, C$ positiv oder jeder negativ, so liegen die Punkte constanten positiven oder negativen Zuwachses auf einem Ellipsoid. Ist $A$ und $B$ positiv, $C$ negativ, so bestimmt die Gleichung

$$
C \cos ^{2} \zeta=A \cos ^{2} \xi+B \cos ^{2} \eta
$$

einen elliptischen Kegel als Ort der Punkte, für welche auch die Incremente zweiter Ordnung verschwinden und daher die dritter Ordnung über Maximum oder Minimum entscheiden müssen; derselbe hüllt asymptotisch das einschalige und zweischalige Hyperboloid ein, auf dem die Punkte constanten positiven und negativen Zuwachses liegen. Das Letztere ist umgekehrt, wenn zwei Coefficienten negativ sind und einer positiv.

Liegen die Punkte $A$ in einer Ebene, so liegen $O$ und zwei der Axen, $x$ und $y$, auch in derselben. Denn in diesem Falle kann durch parallele 
Verschiebung der Grössen $r^{n-1}$ ein geschlossenes Vieleck nur erhalten werden, wenn sie in einer Ebene liegen, und die Transformation von (3.) in (4.) wird erreicht, wenn man $z$ normal zur Ebene nimmt und den Axen $x$ und $y$ in der Ebene eine passende Drehung giebt. Aus (2.) folgt, dass die Summe der Incremente nach zwei auf einander senkrechten Richtungen der Ebene einen stets positiven Werth, nämlich

$$
\varepsilon^{\prime}+\varepsilon^{\prime \prime}=\frac{n^{2}}{2} t^{2} \Sigma r^{n-2},
$$

hat, weshalb absolutes Maximum unmöglich ist, ferner folgt, dass das Increment in normaler Richtung

$$
\frac{n}{2} t^{2} \sum r^{n-2}
$$

beträgt, mithin in dieser Richtung Minimum für positive, Maximum für negative $n$ stattfindet. In Gleichung (4.) ist daher

$$
C=\frac{n}{2} \Sigma r^{n-2}, \quad A+B=\frac{n^{2}}{2} \Sigma r^{n-2} .
$$

Haben $A$ und $B$ verschiedenes Vorzeichen, so liegt die Hauptaxe der Hyperboloide in der Ebene, und in dieser sind zwei Hyperbeln und ihre gemeinschaftlichen Asymptoten der Ort constanten und verschwindenden Zuwachses.

Wenn die Punkte $A$ in einer Geraden liegen, so enthält dieselbe $O$ und eine der Axen, $x$, und es folgt aus Gleichung (2.)

$$
\varepsilon=\frac{n}{2} \Sigma r^{n-2}\left(1+(n-2) \cos ^{2} \xi\right) \text {. }
$$

Daher beträgt der Zuwachs in der Richtung der Geraden

$$
\frac{n(n-1)}{2} t^{2} \Sigma r^{n-2}
$$

so dass in dieser Richtung Maximum nur für $1>n>0$, für alle anderen Werthe von $n$ aber Minimum stattfindet. In normaler Richtung beträgt der Zuwachs

$$
\frac{n}{2} t^{2} \Sigma r^{n-2}
$$

stimmt also im Vorzeichen mit $n$ überein. Für $n<1$ ist der Ort verschwindenden Zuwachses ein Rotationskegel, dessen Axenwinkel $\xi$ durch

$$
\cos \xi=\frac{1}{\sqrt{2-n}}
$$

bestimmt ist, und der die beiden Rotationshyperboloide constanten Zuwachses asymptotisch einhüllt.

3. Einer besonderen Betrachtung bedarf der Fall $n=0$, wo die Gleichung (1.) in 


$$
\Sigma \frac{\cos r t}{r}=0
$$

übergeht und das Maximum oder Minimum des Productes $r_{1} r_{2} r_{3} \ldots$ der Abstände bedingt. Denn mit $\Sigma r^{n}$ ist auch $\Sigma \frac{r^{n}-1}{n}$, folglich, wenn man zur Grenze $n=0$ übergeht, auch $\Sigma \log r$, daher der Logarithmus des Productes der $r$, daher auch dieses Product selbst Minimum oder Maximum. In der That folgt aus

durch Bildung des Productes,

$$
\frac{r^{\prime 2}}{r^{2}}=1-2 t \frac{\cos r t}{r}+\frac{t^{2}}{r^{2}}
$$

$$
\left(\frac{r_{1}^{\prime} r_{2}^{\prime} r_{3}^{\prime} \ldots}{r_{1} r_{2} r_{3} \ldots}\right)^{2}=1-2 t \Sigma \frac{\cos r t}{r}+t^{2}\left[\Sigma \frac{1}{r^{2}}+4 \Sigma \frac{\cos r_{1} t \cdot \cos r_{2} t}{r_{1} r_{2}}\right]-\cdots
$$

Genügt also $O$ den Bedingungen

so hat man

$$
\begin{gathered}
\sum \frac{\cos r t}{r}=0, \\
2 \Sigma \frac{\cos r_{1} t \cdot \cos r_{2} t}{r_{1} r_{2}}=-\Sigma \frac{\cos ^{2} r t}{r^{2}},
\end{gathered}
$$

$$
\left(\frac{r_{1}^{\prime} r_{2}^{\prime} r_{3}^{\prime} \cdots}{r_{1} r_{2} r_{3} \ldots}\right)^{2}=1+t^{2} \Sigma \frac{1-2 \cos ^{2} r t}{r^{2}}+\cdots
$$

daher

$$
\frac{r_{1}^{\prime} r_{2}^{\prime} r_{3}^{\prime} \cdots}{r_{1} r_{2} r_{3} \cdots}=1+\frac{1}{2} t^{2} \Sigma \frac{1-2 \cos ^{2} r t}{r^{2}}+\cdots
$$

Auf den Zuwachs

$$
\text { (5.) } \varepsilon=\frac{1}{2} t^{2} \Sigma \frac{1-2 \cos ^{2} r t}{r^{2}}
$$

lassen sich nun wörtlich die vorhergehenden Betrachtungen anwenden. Die Summe der Incremente nach drei auf einander senkrechten Richtungen ist

$$
\varepsilon^{\prime}+\varepsilon^{\prime \prime}+\varepsilon^{\prime \prime \prime}=\frac{1}{2} t^{2} \Sigma \frac{1}{r^{2}}
$$

also beständig positiv, weshalb kein absolutes Maximum des Productes möglich ist. Bringt man $\varepsilon$ auf die Form der Gleichung (4.), also

$$
\varepsilon=t^{2}\left(A \cos ^{2} \xi+B \cos ^{2} \eta+C \cos ^{2} \zeta\right)
$$

so haben $A, B, C$ die Werthe:

$$
\begin{aligned}
& A=\frac{1}{2} \Sigma \frac{1-2 \cos ^{2} \alpha}{r^{2}}, \\
& B=\frac{1}{2} \Sigma \frac{1-2 \cos ^{2} \beta}{r^{2}}, \\
& C=\frac{1}{2} \Sigma \frac{1-2 \cos ^{2} \gamma}{r^{2}} .
\end{aligned}
$$


Wenn die Punkte $A$ in einer Ebene liegen, so ist der Zuwachs in normaler Richtung

$$
\frac{1}{2} t^{2} \sum \frac{1}{r^{2}}
$$

mithin das Product der Abstände in dieser Richtung stets Minimum. Dagegen beträgt die Summe der Incremente nach zwei senkrechten Richtungen der Ebene stets Null. Daher ist

$$
C=\frac{1}{2} \Sigma \frac{1}{r^{2}}, \quad A+B=0 .
$$

In der Ebene giebt es zwei gleichseitige Hyperbeln und ihre gemeinschaftlichen Asymptoten als Orte constanten und verschwindenden Zuwachses.

Wenn die Punkte $A$ in einer Geraden liegen, so ist

$$
\begin{gathered}
\varepsilon=\frac{1}{2} t^{2} \Sigma \frac{1-2 \cos ^{2} \xi}{r^{2}}, \\
A=-\frac{1}{2} \Sigma \frac{1}{r^{2}}, \quad B=C=\frac{1}{2} \Sigma \frac{1}{r^{2}} .
\end{gathered}
$$

Das Product der Abstände ist also in der Richtung der Geraden Maximum (alle Abstände positiv gerechnet), in jeder Normalen Minimum. Der Zuwachs $\varepsilon$ verschwindet auf einem Rotationskegel, dessen Axenwinkel $45^{\prime \prime}$ beträgt.

\section{\$. 14.}

1. Um die vorstehenden Betrachtungen an einigen Beispielen zu erläutern, bedarf es eines Hilfssatzes:

Wenn die Summe $\Sigma \cos ^{2} r t$ in Bezug auf drei Gerade im Raume oder zwei Gerade einer Ebene denselben Werth hat, so ist sie im Allgemeinen auch in Bezug auf jede Gerade im Raume oder in der Ebene constant. Denn bei der Bezeichnung in $\mathfrak{S}$. 13, 2. kann man $\Sigma \cos ^{2} r t$ durch die Gleichung

$$
\Sigma \cos ^{2} r t=\cos ^{2} \xi \Sigma \cos ^{2} \alpha+\cos ^{2} \eta \Sigma \cos ^{2} \beta+\cos ^{2} \zeta \Sigma \cos ^{2} \gamma^{\prime}
$$

ausdrücken. Ist nun in Bezug auf drei beliebige durch $O$ gehende Gerade $t_{1}, t_{2}, t_{3}$

$$
\begin{aligned}
& c_{1}=\cos ^{2} \xi_{1} \Sigma \cos ^{2} \alpha+\cos ^{2} \eta_{1} \Sigma \cos ^{2} \beta+\cos ^{2} \zeta_{1} \Sigma \cos ^{2} \gamma, \\
& c_{2}=\cos ^{2} \xi_{2} \Sigma \cos ^{2} \alpha+\cos ^{2} \eta_{2} \Sigma \cos ^{2} \beta+\cos ^{2} \zeta_{2} \Sigma \cos ^{2} \gamma, \\
& c_{3}=\cos ^{2} \xi_{3} \Sigma \cos ^{2} \alpha+\cos ^{2} \eta_{3} \Sigma \cos ^{2} \beta+\cos ^{2} \zeta_{3} \Sigma \cos ^{2} \gamma,
\end{aligned}
$$

und bezeichnet $R$ die Determinante der Grössen $\cos ^{2} \xi_{1}, \cos ^{2} \xi_{2}, \ldots$, so ist

$$
R \sum \cos ^{2} \alpha=\left|\begin{array}{lll}
c_{1} & \cos ^{2} \eta_{1} & \cos ^{2} \zeta_{1} \\
c_{2} & \cos ^{2} \eta_{2} & \cos ^{2} \zeta_{2} \\
c_{3} & \cos ^{2} \eta_{3} & \cos ^{2} \zeta_{3}
\end{array}\right| .
$$


Ist $c_{1}=c_{2}=c_{3}=c$ und subtrahirt man von der ersten Verticalreihe der Determinante die beiden folgenden, so erhält man

Mithin ist

$$
\boldsymbol{R} \Sigma \cos ^{2} \alpha=\boldsymbol{c}\left|\begin{array}{ccc}
\cos ^{2} \xi_{1} & \cos ^{2} \eta_{1} & \cos ^{2} \zeta_{1} \\
\cos ^{2} \xi_{2} & \cos ^{2} \eta_{2} & \cos ^{2} \zeta_{2} \\
\cos ^{2} \xi_{3} & \cos ^{2} \eta_{3} & \cos ^{2} \zeta_{3}
\end{array}\right|=\boldsymbol{c} . \boldsymbol{R} .
$$

$$
\Sigma \cos ^{2} \alpha=\Sigma \cos ^{2} \beta=\Sigma \cos ^{2} \gamma=\Sigma \cos ^{2} r t=c=\frac{k}{3},
$$

wenn $k$ die Anzahl der Punkte bezeichnet. Liegen die Punkte $A$ und die Linien $t$ in einer Ebene, so ist

$$
\Sigma \cos ^{2} \alpha=\Sigma \cos ^{2} \beta=c=\frac{k}{2} .
$$

Wenn aber die Determinante $R$ verschwindet, so bleiben $\Sigma \cos ^{2} \alpha$ u.s. w. unbestimmt. Bezeichnen $x_{1}, y_{1}, z_{1}$ die Coordinaten des Púnktes auf $t_{1}$ in der Entfernung der Längeneinheit, so drückt, bei analoger Bedeutung von $x_{2}, y_{2}, \ldots$, die Gleichung

$$
\boldsymbol{R}=\left|\begin{array}{lll}
x_{1}^{2} & y_{1}^{2} & z_{1}^{2} \\
x_{2}^{2} & y_{2}^{2} & z_{2}^{2} \\
x_{3}^{2} & y_{3}^{2} & z_{3}^{2}
\end{array}\right|=0
$$

aus, dass die drei Geraden $t_{1}, t_{2}, t_{3}$ auf einer Kegelfläche zweiten Grades liegen, d. i. auf dem Orte constanter Summe $\Sigma \cos ^{2} r t$. In der Ebene entsprechen ihr zwei Gerade.

2. Der Mittelpunkt $O$ jedes regelmässigen Polyeders mit dessen Eckpunkten $A$ genügt der Bedingung $\Sigma r^{n-1} \cos r t=r^{n-1} \Sigma \cos r t=0$. Denn da die Normalebenen der $r$ das zugeordnete regelmässige Polyeder bilden, so sind die gleichen $r$ den gleichen Flächen dieses Polyeders proportionale Normalen, und es verschwindet daher die Summe ihrer Projectionen auf jede Gerade ${ }^{*}$ ). Bedeutet $k$ die Anzahl der Ecken, so ist in $\$ .13$ Gleichung (2.) $\Sigma \cos ^{2} r t=\frac{k}{3}$ zu setzen, da diese Summe für $t=r_{1}, r_{2}, r_{3}, \ldots$ denselben Werth haben muss, ferner beim Würfel die Normalen der Seitenflächen die drei Hauptrichtungen sind, für welche $\Sigma \cos ^{2} \alpha=\Sigma \cos ^{2} \beta=\Sigma \cos ^{2} \gamma=\frac{8}{3}=\frac{k}{3}$ ist, bei den übrigen Polyedern aber der $\$ .14,1$. angegebene Ausnahmefall unmöglich ist. Man erhält

$$
\varepsilon=\frac{n(n+1)}{6} k t^{2} r^{n-2} \text {. }
$$

*) Vergl. Baltzer Elemente, Trigon. §. 6, 4. 
Für den Fall $n=0$ ergiebt sich aus $\$ .13$ Gleichung (5.)

$$
\varepsilon=\frac{k}{6} \frac{t^{2}}{r^{2}} \text {. }
$$

Im Mittelpunkte $O$ ist daher für $-1<n<0$ die Potenzsumme der Abstände absolutes Maximum, in allen anderen Fällen Minimum, mit Ausnahme von $n=-1$, wo man erst durch das Increment dritter Ordnung über Maximum und Minimum entscheiden kann; das Product der Abstände ist Minimum.

3. Der Mittelpunkt $O$ jedes regelmässigen ebenen Vielecks mit dessen Eckpunkten $A$ erfüllt die Bedingung $\Sigma r^{n-1} \cos r t=r^{n-1} \Sigma \cos r t=0$, da die gleichen Abstände $r$ parallel verschoben ein regelmässiges Vieleck von gleicher Seitenzahl geben. Unter der Voraussetzung, dass $t$ in seiner Ebene bleibt, ist in $\$ .13$ Gleichung (2.) $\Sigma \cos ^{2} r t=\frac{k}{2}$ zu setzen, wodurch sich

$$
\varepsilon=\frac{n^{2}}{4} k t^{2} r^{n-2}
$$

also eine stets positive Grösse ergiebt, weshalb die Potenzsumme der Abstände in der Ebene stets Minimum ist. Um das Increment für ein beliebiges $t \mathbf{z u}$ finden, hat man in $\$ .13$ Gleichung (4.)

$$
\begin{gathered}
A=B=\frac{n^{2}}{4} k r^{n-2}, \\
C=\frac{k n}{2} r^{n-2}
\end{gathered}
$$

zu setzen.

Für $n=0$ verschwindet nach $\$ .13$ Gleichung (5.) in der Ebene auch das Increment zweiter Ordnung. Es ist also $A=B=0, C=\frac{k}{2} \frac{1}{r^{2}}$, der Zuwachs im Raume beträgt

$$
\varepsilon=\frac{k}{2} \frac{t^{2}}{r^{2}} \cos ^{2} \zeta
$$

In der Ebene muss daher das Increment dritter Ordnung über Maximum und Minimum entscheiden. Nun erhält man als Coefficient von $t^{3}$ in der Entwickelung von $\left(\frac{r_{1}^{\prime} r_{2}^{\prime} r_{3}^{\prime} \ldots}{r_{1} r_{2} r_{3} \ldots}\right)^{2}$,

$$
-2 \cdot\left\{\Sigma \frac{\cos r_{1} t}{r_{1}} \frac{1}{r_{2}^{2}}+4 \Sigma \frac{\cos r_{1} t \cdot \cos r_{2} t \cdot \cos r_{3} t}{r_{1} r_{2} r_{3}}\right\},
$$

wo die Summation über alle Combinationen von je zwei und je drei Indices auszudehnen ist. Da das Increment $\delta$ der Wurzel die Hälfte des obigen ist und für $r_{1}=r_{2}=r_{3}=\cdots$ der erste Theil der Summe verschwindet, so hat man

$$
\delta=-4 \frac{t^{3}}{r^{3}} \Sigma \cos r_{1} t \cdot \cos r_{2} t \cdot \cos r_{3} t \text {. }
$$


Man erhält z. B. für das gleichseitige Dreieck, wenn $r_{1} t=u$ gesetzt wird,

$$
\begin{aligned}
\delta & =-4 \frac{t^{3}}{r^{3}} \cos u \cos \left(120^{\prime \prime}+u\right) \cos \left(120^{\prime \prime}-u\right) \\
& =\frac{t^{3}}{r^{3}} \cos u\left(3-4 \cos ^{2} u\right) .
\end{aligned}
$$

Das Product der Abstände, $r_{1} r_{2} r_{3}$, des Mittelpunktes von den Ecken ist daher in den Richtungen $O A_{1}, O A_{2}, O A_{3}$ Maximum, in den ihnen entgegengesetzten Minimum, während in den Richtungen senkrecht zu $O A_{1}, O A_{2}, O A_{3}$ auch das Increment dritter Ordnung verschwindet, so dass um $O$ sechs Felder liegen, für die der Zuwachs abwechselnd positiv und negativ ist.

4. In einem rechtwinkligen Parallelopipedon genügt der Durchschnittspunkt $O$ der Diagonalen der Bedingung $\Sigma r^{n-1} \cos r t=r^{n-1} \Sigma \cos r t=0$ in Bezug auf die drei Normalen der Seitenflächen, also in Bezug auf jede Gerade. Die Hauptaxen $x, y, z$ gehen parallel den Seitenkanten $a, b, c$, und man hat

$$
\begin{gathered}
\cos ^{2} \alpha_{1}=\cos ^{2} \alpha_{2}=\cdots=\frac{a^{2}}{a^{2}+b^{2}+c^{2}}, \\
\Sigma \cos ^{2} \alpha=\frac{8 a^{2}}{a^{2}+b^{2}+c^{2}} .
\end{gathered}
$$

Berücksichtigt man noch die Gleichung: $a^{2}+b^{2}+c^{2}=4 r^{2}$ und die analogen Ausdrücke für $\Sigma \cos ^{2} \beta$ und $\Sigma \cos ^{2} \gamma$, so erhält man

$$
\begin{aligned}
& A=n \cdot r^{n-4}\left((n-1) a^{2}+b^{2}+c^{2}\right), \\
& B=n \cdot r^{n-4}\left(a^{2}+(n-1) b^{2}+c^{2}\right), \\
& C=n \cdot r^{n-4}\left(a^{2}+b^{2}+(n-1) c^{2}\right) .
\end{aligned}
$$

Ob Minimum oder Maximum stattfindet, hängt also, ausser von $n$, auch von dem Grössenverhältnisse der Kanten ab. Für $n=0$ ist

$$
\because \quad A=\frac{16\left(-a^{2}+b^{2}+c^{2}\right)}{\left(a^{2}+b^{2}+c^{2}\right)^{2}},
$$

und analoge Ausdrücke gelten für $B$ und $C$.

5. Im Rechtecke ist $O$ der Durchschnitt seiner Diagonalen, und die Hauptaxen $x$ und $y$ seiner Ehene gehen den Seiten $a$ und $b$ parallel. Man hat daher

$$
\cos ^{2} \alpha_{1}=\cos ^{2} \alpha_{2}=\cdots=\frac{a^{2}}{a^{2}+b^{2}}, \quad \cos ^{2} \beta_{1}=\cdots=\frac{b^{2}}{a^{2}+b^{2}}
$$

zu setzen, wodurch man erhält

$$
\begin{aligned}
& A=\frac{n}{2} r^{n-4}\left(b^{2}+(n-1) a^{2}\right), \\
& B=\frac{n}{2} r^{n-4}\left(a^{2}+(n-1) b^{2}\right) .
\end{aligned}
$$


Für $n=0$, also für das Product der Abstände, ist

$$
A=8 \frac{b^{2}-a^{2}}{\left(a^{2}+b^{2}\right)^{2}}, \quad B=8 \frac{a^{2}-b^{2}}{\left(a^{2}+b^{2}\right)^{3}},
$$

daher der Zuwachs nach beliebiger Richtung der Ebene

$$
\varepsilon=8 \frac{b^{2}-a^{2}}{\left(a^{2}+b^{2}\right)^{2}} t^{2} \cos ^{2} 2 \xi .
$$

6. Wenn man die Normale $O H$ einer bei $O$ rechtwinkligen dreiseitigen Pyramide $O A_{1} A_{2} A_{3}$ rückwärts um $O A_{4}=O H$ verlängert, so erfült $O$ mit den Punkten $A_{1}, A_{2}, A_{3}, A_{4}$ die Bedingung $\Sigma \frac{\cos r t}{r}=0$ in Bezug auf die Geraden $O A_{1}, O A_{2}, O A_{3}$, daher in Bezug auf jede Gerade. Unter Berücksichtigung von:

$$
\frac{1}{r_{4}^{2}}=\frac{1}{r_{1}^{2}}+\frac{1}{r_{2}^{2}}+\frac{1}{r_{3}^{2}}
$$

erhält man als Incremente des Productes der Abstände nach den Richtungen $O A_{1}, O A_{2}, O A_{3}, O A_{4}$,

$$
\begin{aligned}
\varepsilon_{1} & =t^{2}\left(-\frac{1}{r_{1}^{2}}+\frac{1}{r_{2}^{2}}+\frac{1}{r_{3}^{2}}\right), \\
\varepsilon_{2} & =t^{2}\left(\frac{1}{r_{1}^{2}}-\frac{1}{r_{2}^{2}}+\frac{1}{r_{3}^{2}}\right), \\
\varepsilon_{3} & =t^{2}\left(\frac{1}{r_{1}^{2}}+\frac{1}{r_{2}^{2}}-\frac{1}{r_{3}^{2}}\right), \\
\varepsilon_{4} & =-t^{2} r_{4}^{2}\left(\frac{1}{r_{1}^{4}}+\frac{1}{r_{2}^{4}}+\frac{1}{r_{3}^{4}}\right) .
\end{aligned}
$$

Analoges gilt für das Product der Abstände, $O A_{1} \cdot O A_{2} \cdot O A_{3}$, in der Ebene, wenn man die Normale $O H$ eines bei $O$ rechtwinkligen Dreieckes $A_{1} O A_{2}$ rückwärts um $O A_{3}=O H$ verlängert.

\section{\$. 15.}

1. Der Fall, dass die Punkte $A$ auf einer Geraden liegen, bedarf einer besonderen Betrachtung, wenn man den Strecken $\boldsymbol{O A}_{1}, \boldsymbol{O A}_{2}, \ldots$ bei entgegengesetzter Richtung entgegengesetztes Vorzeichen zuschreibt; denn bisher wurden alle $r$ als positiv betrachtet, da, ausser in diesem Falle, zu einer Unterscheidung des Vorzeichens kein Grund vorlag. Wir beschränken uns auf die Betrachtung des Productes

$$
\text { (1.) } O A_{1} . O A_{2} \ldots O A_{k} \text {, }
$$

dessen Minimum - und Maximumpunkte mehrere merkwürdige Eigenschaften besitzen. Dieses Product behält sein Vorzeichen, so lange $O$ in demselben 
Intervalle zwischen zwei Nachbarpunkten $A$ bleibt und erhält, durch Null hindurchgehend, das entgegengesetzte, wenn $O$ in das benachbarte Intervall übergeht. Die Bedingung seines Minimums oder Maximums

$$
\frac{1}{O A_{1}}+\frac{1}{O A_{2}}+\cdots+\frac{1}{O A_{k}}=0
$$

drückt aus, dass in Bezug auf diesen Punkt $O$ der „Mittelpunkt der harmonischen Mittel"*) im Unendlichen liegt. Da nun die Function

$$
X=\frac{1}{O A_{1}-x}+\frac{1}{O A_{2}-x}+\cdots+\frac{1}{O A_{k}-x}
$$

$k-1$ reelle Wurzeln hat, nämlich

$$
\boldsymbol{x}_{1}=\mathrm{OO}_{1}, \quad \boldsymbol{x}_{2}=\mathrm{OO}_{2}, \quad \ldots \quad \boldsymbol{x}_{k-1}=\mathrm{OO}_{k_{-1}-1},
$$

wo die Punkte $O_{1}, O_{2}, \ldots O_{k-1}$ in den Intervallen $A_{1} A_{2}, A_{2} A_{3}, \ldots A_{k-1} A_{k}$ liegen, und da das Product (1.) in den auf einander folgenden Intervallen entgegengesetzte Vorzeichen hat, so hat es in $O_{1}, O_{2}, \ldots O_{k-1}$ abwechselnd Minimum und Maximum.

2. Wenn man in dem Gleichungssysteme

$$
\begin{gathered}
\frac{1}{O_{1} A_{1}}+\frac{1}{O_{1} A_{2}}+\cdots+\frac{1}{O_{1} A_{k}}=0 \\
\frac{1}{O_{2} A_{1}}+\frac{1}{O_{2} A_{2}}+\cdots+\frac{1}{O_{2} A_{k}}=0 \\
\vdots \\
\frac{1}{O_{k-1} A_{1}}+\frac{1}{O_{k-1} A_{2}}+\cdots+\frac{1}{O_{k-1} A_{k}}=0
\end{gathered}
$$

die zweite Gleichung von der ersten abzieht, so erhält man die neue Relation

$$
\frac{1}{O_{1} A_{1} \cdot O_{2} A_{1}}+\frac{1}{O_{1} A_{2} \cdot O_{2} A_{2}}+\cdots+\frac{1}{O_{1} A_{k} \cdot O_{2} A_{k}}=0 \text {. }
$$

Vertauscht man in dieser Gleichung $O_{2}$ mit $O_{3}$ und zieht die erhaltene von der vorstehenden ab, so ergiebt sich

$$
\begin{aligned}
& \frac{1}{O_{1} A_{1} \cdot O_{2} A_{1} \cdot O_{3} A_{1}}+\frac{1}{O_{1} A_{2} \cdot O_{2} A_{2} \cdot O_{3} A_{2}}+\cdots+\frac{1}{O_{1} A_{k} \cdot O_{2} A_{k} \cdot O_{3} A_{k}}=0, \\
& \text { u. s. w. }
\end{aligned}
$$

3. Der Zähler der Function $X$ ist eine Summe von $k$ Gliedern,

$$
\Sigma\left(O A_{2}-x\right)\left(O A_{3}-x\right) \ldots\left(O A_{k}-x\right),
$$

*) „centre des moyennes harmoniques" bei Poncelet, s. Bd. 3, p. 238 dieses Journals. 
wo in jedem Gliede ein Index fehlt. Da seine $k-1$ Wurzeln $O O_{1}, O O_{2}, \ldots$ $O O_{k-1}$ sind, und $x^{k-1}$ den Coefficienten $\pm k$ erhält, so ist bekanntlich

$$
\Sigma\left(O A_{2}-x\right)\left(O A_{3}-x\right) \ldots\left(O A_{k}-x\right)=k\left(O O_{1}-x\right)\left(O O_{2}-x\right) \ldots\left(O O_{k-1}-x\right) .
$$

Hieraus ergeben sich als Coefficientengleichungen die folgenden Relationen zwischen den Abständen der Punkte $O_{1}, O_{2}, \ldots$ und der Punkte $A_{1}, A_{2}, \ldots$ von einem beliebigen Punkte $O$ der Geraden:

$$
\begin{gathered}
\Sigma O A_{2} . O A_{3} \ldots O A_{k}=k . O O_{1} . O O_{2} \ldots O O_{k-1}, \\
2 \Sigma O A_{3} . O A_{4} \ldots O A_{k}=k \Sigma O O_{2} . O O_{3} \ldots O O_{k-1}, \\
3 \Sigma O A_{4} . O A_{5} \ldots O A_{k}=k \Sigma O O_{3} . O O_{4} \ldots O O_{k-1}, \\
\vdots \\
(k-2) \Sigma O A_{k-1} . O A_{k}=k \Sigma O O_{k-2} . O O_{k-1}, \\
(k-1) \Sigma O A_{k}=k \Sigma O O_{k-1} .
\end{gathered}
$$

In diesen Productensummen bedeutet

$$
\sum \boldsymbol{a}_{\boldsymbol{m}+\mathbf{1}} \ldots \boldsymbol{a}_{k}
$$

die Summe aller Producte, in denen $m$ Indices fehlen.

Aus den vorstehenden Gleichungen kann man durch bestimmte Wahl des willkürlichen Punktes $O$ eine grosse Anzahl neuer Relationen herleiten.

Setzt man in der ersten derselben statt $O$ einen der Punkte $A$, etwa $A_{1}$, so erhält man:

$$
A_{1} A_{2} \cdot A_{1} A_{3} \ldots A_{1} A_{k}=k \cdot A_{1} O_{1} \cdot A_{1} O_{2} \ldots A_{1} O_{k-1} .
$$

Aus der letzten Gleichung erhält man:

$$
(k-1)\left(A_{1} A_{2}+A_{1} A_{3}+\cdots+A_{1} A_{k}\right)=k\left(A_{1} O_{1}+A_{1} O_{2}+\cdots+A_{1} O_{k-1}\right) .
$$

Bildet man den letzteren Ausdruck auch für $O=A_{2}, A_{3}, \ldots A_{k}$ und addirł die erhaltenen $k$ Gleichungen, so ergiebt sich:

$$
\left.\begin{array}{c}
A_{1} O_{1}+A_{1} O_{2}+\cdots+A_{1} O_{k-1} \\
+A_{2} O_{1}+A_{2} O_{2}+\cdots+A_{2} O_{k-1} \\
\vdots \\
+A_{k} O_{1}+A_{k} O_{2}+\cdots+A_{k} O_{k-1}
\end{array}\right\}=0
$$

d. h. die Summe der Abstände der sämmtlichen Punkte $O$ von den sämmtlichen Punkten $A$ giebt Null.

In dem einfachsten Falle, dass drei Punkte $A_{1}, A_{2}, A_{3}$ gegeben sind, deren Reihenfolge in positiver Richtung liegt, findet in $O_{1}$ Minimum, in $O_{2}$ Maximum des Productes $O A_{1}, O A_{2} . O A_{3}$ statt. Aus den vorstehenden Glei- 
chungen ergeben sich folgende Relationen:

$$
\begin{aligned}
O A_{2} . O A_{3}+O A_{3} \cdot O A_{1}+O A_{1} \cdot O A_{2} & =30 O_{1} \cdot O O_{2}, \\
2\left(O A_{1}+O A_{2}+O A_{3}\right) & =3\left(O O_{1}+O O_{2}\right) \\
A_{1} A_{2} \cdot A_{1} A_{3} & =3 A_{1} O_{1} \cdot A_{1} O_{2}, \\
A_{2} A_{1} \cdot A_{2} A_{3} & =3 A_{2} O_{1} \cdot A_{2} O_{2}, \\
A_{3} A_{1} \cdot A_{3} A_{2} & =3 A_{3} O_{1} \cdot A_{3} O_{2}, \\
2\left(O_{1} A_{1}+O_{1} A_{2}+O_{1} A_{3}\right) & =3 O_{1} O_{2}, \\
O_{1} A_{1}+O_{1} A_{2}+O_{1} A_{3} & =-\left(O_{2} A_{1}+O_{2} A_{2}+O_{2} A_{3}\right) .
\end{aligned}
$$

§. 16 .

1. Wenn drei Punkte $A_{1}, A_{2}, A_{3}$ gegeben sind, und man mit $r_{1}, r_{2}, r_{3}$ ihre Abstände von $O$, mit $\alpha_{1}, \alpha_{2}, \alpha_{3}$ die Winkel $r_{2} r_{3}, r_{3} r_{1}, r_{1} r_{2}$ bezeichnet, so folgt, weil $r_{1}^{n-1}, r_{2}^{n-1}, r_{3}^{n-1}$ an einander gesetzt ein Dreieck bilden, dessen Winkel die Winkel $\alpha$ zu $180^{\prime \prime}$ ergänzen, die Beziehung

$$
\left.r_{1}^{n-1}: r_{2}^{n-1}: r_{3}^{n-1}=\sin \alpha_{1}: \sin \alpha_{2}: \sin \alpha_{3}^{*}\right) \text {. }
$$

Jeder Abstand $O A$ theilt also den von den beiden anderen eingeschlossenen Winkel in zwei Theile, deren Sinus sich umgekehrt verhalten, wie die $n-1^{\text {ten }}$ Potenzen der anliegenden Abstände.

Die Dreiecksfläche $A_{1} A_{2} A_{3}$ wird in drei Dreiecke zerlegt, deren Verhältniss gleich dem der $n-2^{\text {ten }}$ Potenzen der gegenüberliegenden Abstände ist,

$$
A_{1} O A_{2}: A_{2} O A_{3}: A_{3} O A_{1}=r_{3}^{n-2}: r_{1}^{n-2}: r_{2}^{n-2}
$$

denn es ist z. B. $A_{1} O A_{2}: A_{2} O A_{3}=r_{1} \sin \alpha_{3}: r_{3} \sin \alpha_{1}=r_{3}^{n-2}: r_{1}^{n-2}$.

Verlängert man $O A_{1}, O A_{2}, O A_{3}$, so werden die gegenüberliegenden Dreiecksseiten in Abschnitte getheilt, die sich umgekehrt wie die $n-2^{\text {ten }}$ Potenzen der anliegenden Abstände verhalten; denn schneidet $O A_{3}$ die Seite $A_{1} A_{2}$ in $M_{3}$, so ist $M_{3} A_{1}: M_{3} A_{2}=A_{1} O A_{3}: A_{2} O A_{3}=r_{2}^{n-2}: r_{1}^{n-2}$. Für $n=2$ folgt als bekannte Eigenschaft des Schwerpunktes Gleichheit der Dreiecke $A_{1} O A_{2}, A_{2} O A_{3}, A_{3} O A_{1}$, deren jedes gleich dem Dreieck ist, das $r_{1}, r_{2}, r_{3}$ an einander gesetzt geben, sowie Gleichheit der Abschnitte auf den Seiten.

Für $n=1$ ergiebt sich $\alpha_{1}=\alpha_{2}=\alpha_{3}=120^{\circ}$, indem $r_{1}^{0}, r_{2}^{\prime \prime}, r_{3}^{\prime \prime}$ an einander gesetzt ein gleichseitiges Dreieck geben.

*) s. Steiner in Bd. 13, pag. 362 dieses Journals. 
2. Ist $O$ der Punkt des Minimums oder Maximums für vier Punkte $A_{1}, A_{2}, A_{3}, A_{4}$ im Raume, und setzt man der Grösse und Richtung nach $r_{1}^{n-1}=O B_{1}, r_{2}^{n-1}=B_{1} B_{2}, r_{3}^{n-1}=B_{2} B_{3}, r_{4}^{n-1}=B_{3} O$ an einander, so muss $\mathrm{OA}_{4}$ die Verlängerung von $B_{3} O$ bilden. Da $B_{1} B_{2}$ parallel mit $O A_{2}$ ist, so liegen $r_{1}, r_{2}$ und $B_{1} B_{2}$ in einer Ebene, welche von der Ebene, in welcher $B_{2} B_{3}, B_{3} O, O A_{3}$ und $O A_{4}$, also der Ebene $r_{3} r_{4}$, in der Geraden $O B_{2}$ geschnitten wird. Aus dem Dreieck $B_{1} O B_{2}$ folgt aber, da $\angle B_{1} B_{2} O=\angle A_{2} O B_{2}$ ist,

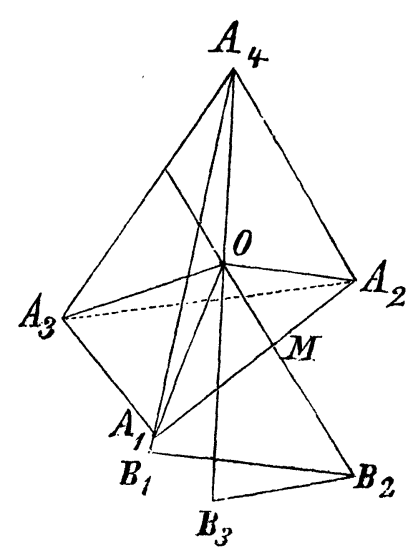

$$
\sin B_{2} O A_{1}: \sin B_{2} O A_{2}=r_{2}^{n-1}: r_{1}^{n-1},
$$

d. h. die Ebene zweier Abstände theilt den Winkel der beiden anderen in zwei Theile, deren Sinus sich umgekehrt verhalten, wie die $n-1^{\text {ten }}$ Potenzen der anliegenden Abstände, für $n=1$ mithin in zwei gleiche Theile.

Ferner theilt jede Ebene durch $O$ und eine Pyramidenkante die gegenüberliegende Pyramidenkante in zwei Abschnitte, die sich umgekehrt verhalten, wie die $n-2^{\text {ten }}$ Potenzen der anliegenden Abstände. Denn schneide $O B_{2}$ die Kante $A_{1} A_{2}$ in $M$, so ist

$$
M A_{1}: M A_{2}=\frac{M A_{1}}{M O}: \frac{M A_{2}}{M O}=\frac{\sin M O A_{1}}{\sin M A_{1} O}: \frac{\sin M O A_{2}}{\sin M A_{2} O}=\frac{r_{2}^{n-1}}{r_{2}}: \frac{r_{1}^{n-1}}{r_{1}}=r_{2}^{n-2}: r_{1}^{n-2}
$$

Der Punkt $O$ ist die gemeinschaftliche Spitze von vier Pyramiden, welche auf den Seitenflächen der gegebenen Pyramide stehen, und deren Verhältniss gleich dem der $n-2^{\text {ten }}$ Potenzen der gegenüberliegenden Abstände ist; z. B.

$$
O A_{1} A_{3} A_{4}: O A_{2} A_{3} A_{4}=M A_{1}: M A_{2}=r_{2}^{n-2}: r_{1}^{n-2} \text {. }
$$

Für $n=2$ haben diese vier Pyramiden gleiches Volumen, und zwar ist jede gleich der Pyramide $O B_{1} B_{2} B_{3}$, welche die $r$ an einander gesetzt bilden. Es ist nämlich allgemein das Volumen $v$ der Pyramide $O B_{1} B_{2} B_{3}$

$$
v=\frac{1}{6} r_{2}^{n-1} r_{3}^{n-1} r_{4}^{n-1} \sin r_{2} r_{3} \sin \widehat{r_{4}} r_{2} r_{3} \text {. }
$$

Das Volumen einer der vier Pyramiden; $v_{1}=O A_{2} A_{3} A_{4}$, ist

$$
\begin{aligned}
& v_{1}=\frac{1}{6} r_{2} r_{3} r_{4} \sin r_{2} r_{3} \sin \widehat{r_{4}} r_{2} r_{3}, \text { daher } \\
& v_{1}=\frac{v}{r_{2}^{n-2} \cdot r_{3}^{n-2} \cdot r_{4}^{n-2}}:
\end{aligned}
$$

Bildet man die analogen Ausdrücke für $v_{2}, v_{3}, v_{4}$, so erhält man ebenfalls 
das oben angegebene Verhältniss derselben, und für $n=2$ folgt, dass $v$ gleich jeder der Pyramiden $v_{1}, v_{2}, v_{3}, v_{4}$ ist.

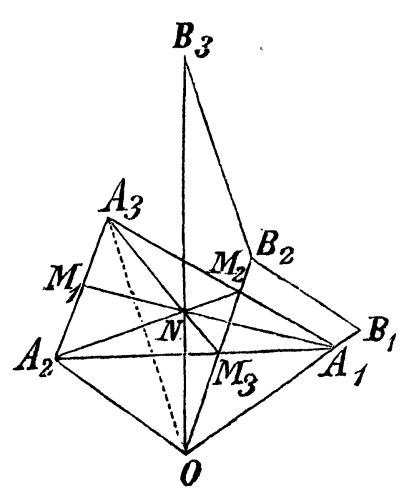

3. Ișt $O$ der Punkt einer gegebenen Ebene, für welchen die Potenzsumme der Abstände von drei ausser ihr liegenden Punkten $A_{1}, A_{2}, A_{3}$ Minimum oder Maximum ist, und setzt man der Grösse und Richtung nach $r_{1}^{n-1}=O B_{1}, r_{2}^{n-1}=B_{1} B_{2}, r_{3}^{n-1}=B_{2} B_{3}$ an einander, so muss $O B_{3}$ eine Normale der Ebene sein. Die Ebene der Geraden $O A_{3}$ und $O B_{3}$ schneidet die Ebene von $O A_{1}$ und $O A_{2}$ in der Geraden $O B_{2}$, durch welche der Winkel $A_{1} O A_{2}$ in zwei Theile getheilt wird, deren Sinusverhältniss ist:

$$
\left.\sin A_{1} O B_{2}: \sin B_{2} O A_{2}=B_{1} B_{2}: O B_{1}=r_{2}^{n-1}: r_{1}^{n-1 *}\right) \text {. }
$$

Schneidet ferner $O B_{2}$ die Dreiecksseite $A_{1} A_{2}$ in $M_{3}$, so folgt, gerade wie im vorigen Falle,

$$
M_{3} A_{1}: M_{3} A_{2}=r_{2}^{n-2}: r_{1}^{n-2} .
$$

Ist endlich $N$ der gemeinschaftliche Durchschnittspunkt von $A_{1} M_{1}, A_{2} M_{2}, A_{3} M_{3}$, so folgt

$$
O N A_{1} A_{3}: O N A_{2} A_{3}=M_{3} A_{1}: M_{3} A_{2}=r_{2}^{n-2}: r_{1}^{n-2} \text {. }
$$

Dies lässt sich folgendermassen zusammenfassen:

Jede Ebene durch die Normale in $O$ und einen Abstand theilt den Winkel der beiden anderen Abstände in zwei Winkel, deren Sinus sich umgekehrt wie die $n-1^{\text {ten }}$ Potenzen, und die gegenüberliegende Dreiecksseite in zwei Abschnitte, die sich umgekehrt wie die $n-2^{\text {ten }}$ Potenzen der anliegenden Abstände verhalten; und die Normale ist gemeinschaftliche Kante dreier Pyramiden, deren Verhältniss gleich dem der $n-2^{\text {ten }}$ Potenzen der gegenüberliegenden Abstände ist.

*) Den Fall $n=1$, wo also die Summe $r_{1}+r_{2}+r_{3}$ ein Minimum ist und der Winkel zweier Abstände von der Ebene des dritten und der Normale halbirt wird, s. Sturm in Gergonne's Annalen Bd. 14, p. 13, und Querret ebendaselbst Bd. 13, p. 329. 
IV. Ueber das Minimum oder Maximum der Potenzsumme der Abstände eines Punktes von Geraden in der Ebene oder Ebenen im Raume.

\$. 17.

1. Bezeichnet $r$ den rechtwinkligen Abstand eines beliebigen Punktes $O$ von einer der gegebenen Geraden oder Ebenen, $r^{\prime}$ den eines anderen Punktes $\boldsymbol{O}^{\prime}$, in der Entfernung $O O^{\prime}=t$, so ist

$$
r^{\prime}=r-t \cos r t,
$$

wobei hinsichtlich des. Vorzeichens die in $\$ .2$ gegebene Regel zu beachten ist. Durch Potenzirung mit einer beliebigen Zahl $n$ folgt nach dem binomischen Satze:

$$
r^{\prime n}=r^{n}-n r^{n-1} t \cos r t+\frac{n . n-1}{1.2} r^{n-2} t^{2} \cos ^{2} r t-\cdots
$$

und hieraus durch Summation

$$
\Sigma r^{\prime n}=\Sigma r^{n}-n t \Sigma r^{n-1} \cos r t+t^{2}\left[\frac{n . n-1}{1.2} \Sigma r^{n-2} \cos ^{2} r t-\cdots\right] .
$$

Da nun, mit Ausschluss des Falles $0<n<1$, das erste Glied der Klammer positiv ist und $t$ so klein genommen werden kann, dass dieses Glied alle folgenden überwiegt, so ist für $\Sigma r^{n-1} \cos r t=0$ der Zuwachs von $\Sigma r^{n}$ ein positiver, mithin $\Sigma r^{n}$ ein Minimum. Dagegen ist für $0<n<1$ das erste Glied der Klammer stets negativ, daher für $\Sigma r^{n-1} \cos r t=0$ auch der Zuwachs von $\Sigma r^{n}$ negativ, $\Sigma r^{n}$ also ein Maximum.

Für den Fall $n=0$ bedingt $\Sigma \frac{\cos r t}{r}=0$ ein Maximum des Productes der Abstände $r$ (vergl. $\$$. 13, 3). Denn aus

$$
\frac{r^{\prime}}{r}=1-t \frac{\cos r t}{r}
$$

folgt durch Bildung des Productes:

$$
\frac{r_{1}^{\prime} r_{2}^{\prime} r_{3}^{\prime} \cdots}{r_{1} r_{2} r_{3} \cdots}=1-t \Sigma \frac{\cos r t}{r}+t^{2} \Sigma \frac{\cos r_{1} t \cdot \cos r_{2} t}{r_{1} r_{2}}-\cdots
$$

Unter der Bedingung dass

$$
\begin{gathered}
\Sigma \frac{\cos r t}{r}=0, \\
\sum \frac{\cos ^{2} r t}{r^{2}}=-2 \Sigma \frac{\cos r_{1} t \cdot \cos r_{2} t}{r_{1} r_{2}}
\end{gathered}
$$


erhält man

$$
\frac{r_{1}^{\prime} r_{2}^{\prime} r_{3}^{\prime} \cdots}{r_{1} r_{2} r_{3} \cdots}=1-\frac{1}{2} t^{2} \Sigma \frac{\cos ^{2} r t}{r^{2}}+\cdots
$$

Da also der Zuwachs beständig negativ ist, so ist das Product $r_{1} r_{2} r_{3} \ldots$ Maxinuum.

Berücksichtiget man noch, dass die Bedingungsgleichung $\Sigma r^{n-1} \cos r t=0$ dieselbe ist, wie die in $\$ \mathbf{1 3}, 1$. erhaltene, so hat man folgendes Resultat:

Ist für einen Punkt $O$ die Summe der Projectionen der $n-1^{\text {ten }}$ Potenzen seiner Abstände von Geraden oder Ebenen in Bezug auf jede Gerade gleich Null, so ist die Summe der $n^{\text {ten }}$ Potenzen dieser Abstände im Falle $0<n<1$ ein Maximum, für alle anderen Werthe von $n$ ein Minimum. Für denselben Punkt $O$ ist auch die Summe der $n^{\text {ten }}$ Potenzen seiner Abstände von den Fusspunkten jener Senkrechten Minimum oder Maximum. Endlich ist im Falle $n=0$ das Product der Abstånde des Punktes $O$ von den Geraden oder Ebenen ein Maximum, das Product der Abstände von den Fusspunkten jener Abstände Minimum oder Maximum (s. $\$$. 13, 3).

Für denselben Punkt $O$ ist bei Geraden in der Ebene auch die Potenzsumme der nicht rechtwinklig, sondern unter einem beliebigen Winkel $u$ genommenen Abstände $\varrho$ Maximum oder Minimum, sowie auch die seiner $\mathbf{A b}$ stände von den Endpunkten der $\varrho$ auf den Geraden. Denn mit $\Sigma r^{n}$ ist auch $\Sigma \varrho^{n}=\frac{\Sigma r^{n}}{\cos ^{n} u}$ Minimum oder Maximum; und bildet die durch $\boldsymbol{O}$ gelegte Gerade $\tau$ mit $t$ ebenfalls den Winkel $u$, so ist mit $\Sigma r^{n-1} \cos r t=0$ die Gleichung $\Sigma \varrho^{n-1} \cos \varrho \tau=0$ identisch. Dreht man also das System der gegebenen Geraden in seiner Ebene um den Punkt $O$, so wird es von dem festen Strahlenbüschel der $r$ beständig in Punkten geschnitten, für welche die Potenzsumme der Abstände von $O$ Minimum oder Maximum ist.

Liegt $O$ auf einer vorgeschriebenen Geraden oder Ebene, so muss in Bezug auf diese Gerade oder in Bezug auf jede Gerade, dieser Ebene $\Sigma r^{n-1} \cos r t=0$ sein.

2. Aus $\Sigma r^{n-1} \cos r t=0$ ergiebt sich eine bemerkenswerthe Relation. Multiplicirt man die Gleichung $r^{\prime}=r-t \cos r t$ mit $r^{n-1}$, so erhält man durch Summation

$$
\Sigma r^{\prime} \cdot r^{n-1}=\Sigma r^{n}
$$

Mithin ist für jeden beliebigen Punkt $O^{\prime}$ die Summe $\Sigma r^{\prime} . r^{n-1}$ constant (mit 
Ausschluss des Falles $n=1$ ). Hat man z. B. ein Vieleck, das einem Kreise, oder ein Polyeder, das einer Kugel mit dem Halbmesser $a$ umschrieben ist, so erhält man, wenn man $O^{\prime}$ zum Mittelpunkt nimmt, also $r^{\prime}=a$ setzt, $a \Sigma r^{n-1}=\Sigma r^{n}$; es ist also für jedes $n$ der Quotient $\Sigma r^{n}: \Sigma r^{n-1}$ constant, nämlich gleich dem Halbmesser. Schneiden sich sämmtliche Geraden oder Ebenen mit Ausnahme der ersten in einem Punkte im senkrechten Abstande $h$ von dieser, so ist

$$
h \cdot r_{1}^{n-1}=\Sigma r^{n} .
$$

3. Schneidet $O O^{\prime}$ die gegebenen Geraden oder Ebenen in den Punkten $P_{1}, P_{2}, \ldots$, so ist $\cos r t=\frac{r}{O P}$, mithin kann man statt $\Sigma r^{n-1} \cos r t=0$ auch schreiben

$$
\Sigma \frac{r^{n}}{O P}=0
$$

welche Summe also beständig Null bleibt, wenn man die schneidende Gerade beliebig um den Punkt $O$ dreht ${ }^{*}$ ). Ist $n=0$, also für $O$ das Product der Abstände ein Maximum, so folgt $\Sigma \frac{1}{O P}=0$ (s. \$. 15). Diese letztere Relation gilt z. B. für jede Gerade durch den Mittelpunkt eines regelmässigen Vielecks, durch die Mitte der Diagonale eines Vierecks, zu welcher dasselbe symmetrisch ist, durch den Schwerpunkt eines Dreiecks oder einer dreiseitigen Pyramide (s. den folgenden Paragraphen).

4. Im Falle $n=1$ giebt es keinen Punkt $O$ des Minimums oder Maximums, sondern, wie bekannt ${ }^{* *}$ ), giebt es im Allgemeinen für gegebene Gerade parallele Gerade und für gegebene Ebenen parallele Ebenen von der Eigenschaft, dass für jeden Punkt einer solchen die Summe der Abstände, $\Sigma r$, constant bleibt. Denn in der Gleichung

$$
\Sigma r^{\prime}=\Sigma r-t \Sigma \cos r t
$$

findet entweder der besondere Fall statt, dass $\Sigma \cos r t$ in Bezug auf jede beliebige Gerade $t$ verschwindet, daher $\Sigma r$. für jeden Punkt constant ist, wie z. B. bei einem gleichseitigen Vieleck; oder es lässt sich eine Richtung paralleler Geraden oder eine Stellung paralléler Ebenen angeben, für die $\Sigma \cos r t=0$ ist.

*) s. Poncelet in Band 3,'p. 241 dieses Journals. Der „Mittelpunkt der harmonischen Mittel" liegt also in Bezug auf $O$ im Unendlichen, wenn $\frac{1}{O P_{1}}, \therefore$ die Coefficienten $r_{1}^{n}, \ldots$ erhalten.

**). Vergl. Baltzer Elemente, Plan. §. 10, 8. und Stereometrie §.8, 16. 
§. 18.

1. Wenn drei Gerade $a_{1}, a_{2}, a_{3}$ sich in den Punkten $A_{3}, A_{1}, A_{2}$ schneiden, und $M_{1}$ der Punkt der Geraden $a_{1}$ ist, für welchen die Potenzsumme der Abstände von $a_{2}$ und $a_{3}$ Minimum oder Maximum ist, so hat man die Bedingungsgleichung

$$
r_{2}^{n-1} \cos r_{2} a_{1}+r_{3}^{n-1} \cos r_{3} a_{1}=0
$$

folglich

$$
\begin{gathered}
r_{2}^{n-1}: r_{3}^{n-1}=\sin A_{2}: \sin A_{3}=a_{2}: a_{3}, \\
M_{1} A_{2}: M_{1} A_{3}=\frac{r_{3}}{\sin A_{2}}: \frac{r_{2}}{\sin A_{3}}=a_{3}^{\frac{n}{n-1}}: a_{2}^{\frac{n}{n-1}}=r_{3}^{n}: r_{2}^{n}
\end{gathered}
$$

Die Abschnitte der Geraden $a_{1}$ verhalten sich demnach wie die $\frac{n}{n-1}$ ten Potenzen der anliegenden Dreiecksseiten. Zur Bestimmung von $r_{2}$ und $r_{3}$ sei noch die Fläche $f$ des Dreiecks $A_{1} A_{2} A_{3}$ gegeben, so hat man $2 f=a_{2} r_{2}+a_{3} r_{3}$, und folglich

$$
r_{2}=\frac{2 f \cdot a_{2}^{\frac{1}{n-1}}}{a_{2}^{\frac{n}{n-1}}+a_{3}^{\frac{n}{n-1}}}, \quad r_{3}=\frac{2 f \cdot a_{3}^{\frac{1}{n-1}}}{a_{2}^{\frac{n}{n-1}}+a_{3}^{\frac{n}{n-1}}} .
$$

2. Ist $O$ der Punkt des Minimums oder Maximums dreier Geraden $a_{1}, a_{2}, a_{3}$, so folgt, weil das Dreieck, welches $r_{1}^{n-1}, r_{2}^{n-1}, r_{3}^{n-1}$ an einander gesetzt geben, mit dem gegebenen Dreiecke gleichwinklig, ihm also ähnlich ist,

$$
r_{1}^{n-1}: r_{2}^{n-1}: r_{3}^{n-1}=a_{1}: a_{2}: a_{3} .
$$

Hieraus ergeben sich in Verbindung mit $2 f=a_{1} r_{1}+a_{2} r_{2}+a_{3} r_{3}=a_{1} h_{1}=a_{2} h_{2}=a_{3} h_{3}$, wo $h_{1}, h_{2}, h_{3}$ die Höhen des Dreiecks bezeichnen, als Werthe der $r$ :

$$
\begin{aligned}
r_{1} & =h_{1} \frac{a_{1}^{\frac{n}{n-1}}}{a_{1}^{\frac{n}{n-1}}+a_{2}^{\frac{n}{n-1}}+a_{3}^{\frac{n}{n-1}}}, \\
r_{2} & =h_{2} \frac{a_{2}^{\frac{n}{n-1}}}{a_{1}^{\frac{n}{n-1}}+a_{2}^{\frac{n}{n-1}}+a_{3}^{\frac{n}{n-1}}}, \\
r_{3} & =h_{3} \frac{a_{3}^{\frac{n}{n-1}}}{a_{1}^{\frac{n}{n-1}}+a_{2}^{\frac{n}{n-1}}+a_{3}^{\frac{n}{n-1}}} .
\end{aligned}
$$


Als Minimalsumme folgt

$$
r_{1}^{n}+r_{2}^{n}+r_{3}^{n}=\frac{2^{n} \cdot f^{n} .}{\left(a_{1}^{\frac{n}{n-1}}+a_{2}^{\frac{n}{n-1}}+a_{3}^{\frac{n}{n-1}}\right)^{n-1}} .
$$

Aus der allgemeinen Gleichung $\Sigma \frac{r^{n}}{O P}=0(\$ .17,3$.$) folgt, wenn man die$ Gerade $t$ parallel einer Dreiecksseite $A_{2} A_{3}$ legt, abgesehen vom Vorzeichen,

$$
O P_{3}: O P_{2}=r_{3}^{n}: r_{2}^{n}=M_{1} A_{2}: M_{1} A_{3} .
$$

Daher liegt $O$ auf der Geraden $A_{1} M_{1}$. Mithin bestimmt sich $O$ als der gemeinschaftliche Durchschnittspunkt dreier Geraden $A_{1} M_{1}, A_{2} M_{2}, A_{3} M_{3}$, jede von einer Ecke des Dreiecks nach dem Minimum-(Maximum) Punkte der gegenüberliegenden Seite. Dies folgt auch aus der Bemerkung, dass, vermöge der oben angegebenen Werthe von $r_{1}, r_{2}, r_{3}, O$ der Schwerpunkt der Punkte $A_{1}, A_{2}, A_{3}$ ist, wenn jeder als Gewicht die Potenz $\frac{n}{n-1}$ der gegenüberliegenden Dreiecksseite hat; $M_{1}, M_{2}, M_{3}$ sind dann die Schwerpunkte je zweier Punkte $A$. Für $n=0$ ist $O$ der Schwerpunkt der gleich schweren Punkte $A_{1}, A_{2}, A_{3}$; für jede durch denselben gelegte Gerade gilt daher die Relation

$$
\left.\frac{1}{O P_{1}}+\frac{1}{O P_{2}}+\frac{1}{O P_{3}}=0^{*}\right), \quad(\$ .17,3 .),
$$

und ferner ist das Product seiner Abstände von den Dreiecksseiten ein Maximum, das der Abstände von den Fusspunkten jener relatives Minimum oder Maximum.

Sind $S_{1}, S_{2}, S_{3}$ die Fusspunkte von $r_{1}, r_{2}, r_{3}$ oder auch die Durchschnittspunkte der unter beliebigem Winkel genommenen Entfernungen mit den Seiten, so ist (nach $\$$. 17, 1.) $O$ zugleich der Punkt des Minimums oder Maximums für $S_{1}, S_{2}, S_{3}$, folglich nach $\$$. 16, 1 :

$$
S_{1} O S_{2}: S_{2} O S_{3}: S_{3} O S_{1}=r_{3}^{n-2}: r_{1}^{n-2}: r_{2}^{n-2}=a_{3}^{\frac{n-2}{n-1}}: a_{1}^{\frac{n-2}{n-1}}: a_{2}^{\frac{n-2}{n-1}} \text {. }
$$

Für $n=0$ folgt, dass das Verhältniss der Dreiecke, deren gemeinschaftliche Spitze der Schwerpunkt und deren Ecken die Projectionen desselben auf die Dreiecksseiten sind, gleich dem Verhältnisse der Quadrate der gegenüberliegenden Dreiecksseiten ist. Ist $O$ der Schwerpunkt eines rechtwinkligen

*) s. Müller in Grunerts Archiv 16, p. 1. 
Dreiecks, so ist das der Hypotenuse gegenüberliegende Dreieck gleich der Summe der beiden anderen.

3. Ist $O_{4}$ der Punkt einer Ebene $f_{4}$, für welchen die Potenzsumme der Abstände von drei Ebenen $f_{1}, f_{2}, f_{3}$ Minimum oder Maximum ist, so muss er der Bedingung genügen

$$
r_{1}^{n-1} \cos r_{1} t+r_{2}^{n-1} \cos r_{2} t+r_{3}^{n-1} \cos r_{3} t=0 .
$$

Schneiden sich diese vier Ebenen in den Punkten $A_{1}, A_{2}, A_{3}, A_{4}$, die den Ebenen von gleichem Index gegenüber liegen, und nimmt man als $t$ eine durch $O_{4}$ parallel mit $A_{2} A_{3}$ gelegte Gerade, welche $A_{1} A_{3}$ in $F, A_{1} A_{2}$ in $G$ schneidet, so geht obige Gleichung in die einfachere $r_{2}^{n-1} \cos \alpha_{2}=r_{3}^{n-1} \cos \alpha_{3}$ über, wo $\alpha_{2}$ und $\alpha_{3}$ die spitzen Winkel bedeuten, die $r_{2}$ und $r_{3}$ mit $F G$ bilden. Wird ferner $A_{2} A_{3}$ von $A_{1} O_{4}$ in $M_{1}$ geschnitten, so hat man

$$
A_{1} A_{3} A_{4} O: A_{1} A_{2} A_{4} O=f_{2} r_{2}: f_{3} r_{3}=M_{1} A_{3}: M_{1} A_{2}=O_{4} F: O_{4} G=\frac{r_{2}}{\cos \alpha_{2}}: \frac{r_{3}}{\cos \alpha_{3}} \text {. }
$$

Hieraus ergiebt sich in Verbindung mit der vorhergehenden Gleichung:

$$
\begin{gathered}
f_{2}: f_{3}=r_{2}^{n-1}: r_{3}^{n-1}, \\
M_{1} A_{3}: M_{1} A_{2}=f_{2}^{\frac{n}{n-1}}: f_{3}^{\frac{n}{n-1}} .
\end{gathered}
$$

Demnach bestimmt sich der Punkt $O_{4}$ als der gemeinschaftliche Durchschnittspunkt dreier Geraden $A_{1} M_{1}, A_{2} M_{2}, A_{3} M_{3}$, jede von einer Ecke des Dreiecks nach dem Punkte der gegenüberliegenden Seite, welcher dieselbe im Verhältniss der $\frac{n}{n-1}^{\text {ten }}$ Potenzen der anstossenden Pyramidenflächen theilt, d. i. nach dem Punkte des Minimums oder Maximums dieser Pyramidenkante in Bezug auf die anstossenden beiden Flächen.

4. Es ist nun aber leicht zu sehen, dass für jeden Punkt der Geraden $A_{4} O_{4}$ in Bezug auf eine zur Ebene $f_{4}$ parallele Gerade $t$ die Bedingung

$$
r_{1}^{n-1} \cos r_{1} t+r_{2}^{n-1} \cos r_{2} t+r_{3}^{n-1} \cos r_{3} t=0,
$$

folglich auch, wegen $r_{+} t=90^{\circ}$,

$$
r_{1}^{n-1} \cos r_{1} t+r_{2}^{n-1} \cos r_{2} t+r_{3}^{n-1} \cos r_{3} t+r_{4}^{n-1} \cos r_{4} t=0
$$

erfüllt ist, dass mithin der Minimumpunkt $O$ auf der Geraden $A_{4} O_{4}$ liegen muss. Daher ergiebt sich der Punkt $O$ für vier Ebenen als der gemeinschaftliche Durchschnittspunkt der vier Geraden $\mathrm{A}_{1} \mathrm{O}_{1}, \mathrm{~A}_{2} \mathrm{O}_{2}, \mathrm{~A}_{3} \mathrm{O}_{3}, \mathrm{~A}_{4} \mathrm{O}_{4}$, jede von einer Ecke der Pyramide nach dem Punkte des Minimums (oder Maximums) der gegenüberliegenden Seitenfläche. Die $n-1^{\text {ten }}$ Potenzen seiner Abstände sind 
den zugehörigen Seitenflächen proportional,

$$
r_{1}^{n-1}: r_{2}^{n-1}: r_{3}^{n-1}: r_{4}^{n-1}=f_{1}: f_{2}: f_{3}: f_{4} .
$$

Nimmt man hierzu die Gleichung

$$
3 v=f_{1} r_{1}+f_{2} r_{2}+f_{3} r_{3}+f_{4} r_{4}=f_{1} h_{1},
$$

wo $v$ das Volumen und $h_{1}$ die zu $f_{\mathfrak{l}}$ gehörige Höhe der Pyramide bezeichnet, so erhält man

$$
r_{1}=\frac{3 v \cdot f_{1}^{\frac{1}{n-1}}}{f_{1}^{\frac{n}{n-1}}+f_{2}^{\frac{n}{n-1}}+f_{3}^{\frac{n}{n-1}}+f_{4}^{\frac{n}{n-1}}}=h_{1} \frac{f_{1}^{\frac{n}{n-1}}}{f_{1}^{\frac{n}{n-1}}+f_{2}^{\frac{n}{n-1}}+f_{3}^{\frac{n}{n-1}}+f_{4}^{\frac{n}{n-1}}} .
$$

Analoge Ausdrücke gelten für die übrigen $r$; als Minimalsumme folgt hieraus

$$
r_{1}^{n}+r_{2}^{n}+r_{3}^{n}+r_{4}^{n}=\frac{3^{n} \cdot v^{n}}{\left(f_{1}^{\frac{n}{n-1}}+f_{2}^{\frac{n}{n-1}}+f_{3}^{\frac{n}{n-1}}+f_{4}^{\frac{n}{n-1}}\right)^{n-1}} .
$$

Die Werthe der Abstände $r_{1}, r_{2}, r_{3}, r_{4}$ zeigen, dass $O$ der Schwerpunkt der vier Durchschnittspunkte der gegebenen Ebenen ist, wenn jeder als Gewicht die $\frac{n}{n-1}^{\text {te }}$ Potenz der gegenüberliegenden Seitenfläche hat. Die Punkte $O_{1}, O_{2}, O_{3}, O_{4}$ sind dann die Schwerpunkte je dreier, die Punkte $M$ die Schwerpunkte je zweier der Punkte $A$. Für $n=0$ ist also $O$ der Schwerpunkt der gleich schweren Punkte $A$. Für jede durch ihn gelegte Gerade, welche die Flächen der Pyramide in $P_{1}, P_{2}, P_{3}, P_{4}$ schneidet, gilt daher die Relation (s. \$. 17, 3.)

$$
\left.\frac{1}{O P_{1}}+\frac{1}{O P_{2}}+\frac{1}{O P_{3}}+\frac{1}{O P_{4}}=0^{*}\right)
$$

ferner ist das Product seiner senkrechten Abstände von den Seitenflächen ein Maximum und das seiner Abstände von den Fusspunkten dieser Senkrechten Minimum oder Maximum.

Der Punkt $O$ ist der Scheitel von vier Pyramiden, die sich wie die $\frac{n}{n-1}^{\text {ten }}$ Potenzen der Seitenflächen der gegebenen Pyramide verhalten, auf denen sie stehen, wie z. B.

$$
O A_{1} A_{4} A_{3}: O A_{1} A_{4} A_{2}=M_{1} A_{3}: M_{1} A_{2}=f_{2}^{\frac{n}{n-1}}: f_{3}^{\frac{n}{n-1}} \text {. }
$$

Nach \$. 17, 1. ist $O$ aber auch Punkt des Minimums oder Maximums für die

*) s. Mïller in Grunerts Archiv 16, p. 1. 
Fusspunkte $S_{1}, S_{2}, S_{3}, S_{4}$ der Senkrechten $r$. Da dieselben die Eckpunkte von vier Pyramiden mit der gemeinschaftlichen Spitze $O$ bilden, die sich nach \$. 16, 2. wie die $n-2^{\text {ten }}$ Potenzen der gegenüberliegenden $r$ verhalten, so hat man

$$
O S_{1} S_{2} S_{3}: O S_{1} S_{2} S_{4}=r_{4}^{n-2}: r_{3}^{n-2}=f_{4}^{\frac{n-2}{n-1}}: f_{3}^{\frac{n-2}{n-1}}
$$

Für $n=0$ folgt:

Die vier dreiseitigen Pyramiden, deren gemeinschaftliche Spitze der Schwerpunkt einer dreiseitigen Pyramide und deren übrigen Ecken die Projectionen des Schwerpunktes auf die Seitenflächen sind, verhalten sich wie die Quadrate der gegenüberliegenden Seitenflächen.

Leipzig, 1863. 